\title{
STRUCTURE THEORY OF FAITHFUL RINGS II. RESTRICTED RINGS $\left({ }^{1}\right)$
}

\author{
BY \\ R. E. JOHNSON
}

The first paper of this series $\left({ }^{2}\right)$ concerned itself mainly with closure operations on a lattice. This paper applies these results to the global structure theory of a faithful ring and its modules.

A ring $R$ is called faithful if $a R=0$ implies $a=0$. Let $\mathscr{L}\left(\mathcal{L}^{\natural}\right)$ be the lattice of right (two-sided) ideals of $R$, and $F^{\prime \prime}=\left\{A ; A \in \mathcal{L}^{\iota}, A \cap A^{l}=0, A=A^{l l}\right\}$. The set $\mathcal{F}^{\prime \prime}$ becomes a Boolean algebra with the obvious definition of the union operation. In case $\mathcal{F}^{\prime \prime}$ is complete, $R$ is called a restricted ring. Being complete, $\mathcal{F}^{\prime \prime}$ induces a closure operation $f$ on $\mathscr{L}$ and $\mathfrak{L}^{\mathfrak{}}$. It is shown that if $f$ is homogeneous, then there exist irreducible rings $A_{i}$ such that $\sum_{i} A_{i} \subset R$ $\subset \sum_{i}^{*} \bar{A}_{i}$, where $\sum\left(\sum^{*}\right)$ designates the discrete (full) direct sum and $\bar{A}_{i}$ is a universal extension ring of $A_{i}$. A reducible ring is shown to be restricted, where $R$ is reducible if and only if for every pair $A, B$ of ideals of $R$ with zero intersection, there exist ideals $A^{\prime} \supset A$ and $B^{\prime} \supset B$ also with zero intersection such that $A^{\prime \prime} \cap B^{\prime l}=0$.

Modules of a faithful ring are studied in the fourth section. It is shown that every suitably restricted closure operation on $\mathscr{L}$ induces a closure operation on $\mathfrak{N}$, the lattice of submodules of a $R$-module $M$. If ${ }^{l} A, A \subset R$, designates the annihilator of $A$ in $M$, then it is shown in the fifth section that $\mathcal{H}=\left\{{ }^{l} A\right.$; $\left.A \in \mathcal{F}^{\prime \prime}\right\}$ is a Boolean algebra.

In the final two sections, it is assumed that the ring $R$ and the $R$-module $M$ have the property that for every nonzero element $x$ in $R$ or $M$ there exists a nonzero $A \in \mathcal{L}$ such that $x a \neq 0$ for every nonzero $a \in A$. It is shown that $R$ is restricted, and that every $A$ in $\mathscr{L}(\mathfrak{T})$ has a unique maximal essential extension $A^{*}\left(A^{t}\right)$. For the closure operation $s$ on $\mathscr{L}$ so defined, $\mathfrak{F}^{\prime \prime}$ is proved to be the center of the lattice $\mathscr{L}^{s}$, and similarly for $\mathcal{H}$ in $\mathfrak{M}^{t}$. Imbedding $M$ in its unique minimal injective extension $\hat{M}$, it is proved that the lattice $\mathbb{N}^{t}$ is isomorphic to the lattice of principal right ideals of the centralizer $\mathfrak{C}$ of $R$ over $\hat{M}$. If $s$ is atomic, $\mathfrak{C}$ is a full direct sum of primitive rings with minimal right ideals.

1. Faithful rings. If $R$ is a ring, then $\mathscr{L}(R)\left(\mathscr{L}^{\mathfrak{l}}(R)\right)$ will designate the lattice of all right (two-sided) ideals of $R$. We shall upon occasion write $\mathscr{L}$ or $\mathcal{L}^{l}$ if the ring in question is obvious. For each subset $A$ of $R, A^{l}\left(A^{r}\right)$ will

Presented to the Society, September 2, 1955; received by the editors June 6, 1956.

(1) This research was supported in part by a grant from the National Science Foundation.

(2) Structure theory of faithful rings, I. Closure operations on lattices. This paper is referred to henceforth as (FI). The bibliography of both papers is contained at the end of (FI). 
designate the left (right) annihilator of $A$ in $R$. Clearly $A^{r} \in \mathcal{L}$ for every $A \subset R$, and $A \cdot \in \mathscr{L}^{\prime}$ if $A \in \mathscr{L}$. The mapping $l r: A \rightarrow A^{l r}$ is a closure operation on both $\mathfrak{L}$ and $\mathscr{L}$.

A ring $R$ will be called (left) faithful if $R^{l}=0$. One could define right faithful rings analogously. We shall investigate in this section the general properties of faithful rings. Thus each ring $R$ considered in this section is assumed to be faithful.

The ideals $S$ of $R$ for which $S^{l}=0$ play an important part in our discussion. Let us designate by $J(R)$ the set of all such ideals. By assumption, $R \in J(R)$; and if $S, T \in J(R)$, then $S T \in J(R)$. Thus it is evident that $\Im(R)$ is a (not necessarily complete) sublattice of $\mathscr{L}^{\mathfrak{}}(R)$.

If $A \in \mathfrak{L}(R), A$ is called prime [10, $\S 2]$ if and only if

$$
r S \subset A, \text { for } r \in R \text { and } S \in \Im(R) \text {, implies } r \in A \text {. }
$$

We shall designate by $\mathcal{L}^{p}(R)$ the set of all prime right ideals of $R$. It is proved in (FI, §5) that $p \in C_{m}^{0}(\mathscr{L})$ and that $p \leqq l r$.

If $A \in \mathscr{L}^{p}(R)$ and $B$ is the bound of $A$, so that $B$ is the largest ideal of $R$ contained in $A$, then $B \in \mathcal{L}^{p}(R)$ also. Thus, if $r S \subset B$ for some $r \in R$ and $S \in J(R)$, then $(r) S \subset B$ and $(r) \subset A$, where $(r)$ designates the ideal of $R$ generated by $r$. Clearly, then, $(r) \subset B$ and $B \in \mathcal{L}^{p}(R)$. Consequently, for each

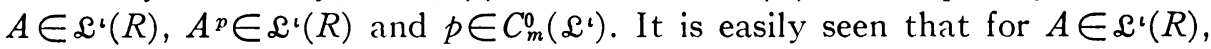
$A^{p}=R$ if and only if $A \in \Im(R)$.

We shall need to consider those ideals of $R$ that as rings are faithful. We separate such ideals into three classes as follows:

$$
\begin{aligned}
\mathscr{F}(R) & =\left\{A ; A \in \mathcal{L}^{\imath}(R), A \cap A^{l}=0\right\}, \\
\mathcal{F}^{\prime}(R) & =\left\{A ; A, A^{l} \in \mathcal{F}(R)\right\}, \\
\mathcal{F}^{\prime \prime}(R) & =\left\{A ; A \in \mathscr{F}(R), A=A^{\imath l}\right\} .
\end{aligned}
$$

Clearly $\mathcal{F}^{\prime \prime}(R) \subset \mathcal{F}^{\prime}(R) \subset \mathfrak{F}(R)$. Also

$$
A^{l} \subset A^{r}, \quad A \subset A^{l l} \quad \text { for each } A \in \mathcal{F}(R) .
$$

It is evident that $0, R \in \mathcal{F}^{\prime \prime}(R)$, that $\Im(R) \subset \mathcal{F}^{\prime}(R)$, and that if $A \in \mathcal{F}^{\prime}(R)$ then $A \in \Im\left(A^{l l}\right)$ and $A^{l} \in \mathcal{F}^{\prime \prime}(R)$. Also

$A \cap B=0$ if and only if $A B=0 ; \quad A \in \mathscr{L}(R)$ and $B \in \mathcal{F}(R)$.

If $r \in R, S \in \mathcal{J}(R)$, and $A \in \mathcal{F}(R)$, and if $r S \subset A^{l}$, then $r A S \subset A \cap A^{l}=0$ and $r A=0$. Thus $r \in A^{l}$, and we conclude that $A^{l} \in \mathfrak{L}^{p}(R)$. In particular, $\mathcal{F}^{\prime \prime}(R)$ $\subset \mathfrak{L}^{p}(R)$.

1.1. Lemma. If $A \in \mathscr{L}^{\prime}(R)$ and $B \in \mathcal{F}(R)$, then $(A \cap B)^{l} \cap B=A^{\prime} \cap B$.

Proof. Clearly $(A \cap B)^{l} \cap B \supset A^{l} \cap B$. Since $\left[(A \cap B)^{l} \cap B\right] A B \subset\left[(A \cap B)^{l}\right.$ $\cap B](A \cap B)=0$, we have $\left[(A \cap B)^{l} \cap B\right] A \subset B^{l} \cap B=0$. Hence $(A \cap B)^{l} \cap B$ $\subset A^{\prime} \cap B$, and 1.1 is proved. 
If, in 1.1, also $A \in \mathcal{F}(R)$, then $(A \cap B)^{l} \cap(A \cap B)=A \cap A^{l} \cap B=0$, and $A \cap B \in \mathcal{F}(R)$. If $A, B \in \mathcal{F}^{\prime \prime}(R)$, then so is $A \cap B$ since $A \cap B \subset(A \cap B)^{\imath l}$ $\subset A^{l l} \cap B^{l l}$. We have proved the following theorem.

1.2. THEOREM. Both $\mathcal{F}(R)$ and $\mathcal{F}^{\prime \prime}(R)$ are closed under the finite intersection operation.

The F-sets are not generally closed under the union operation, although we do have the following result.

1.3. Theorem. If $\left\{A_{i}\right\} \subset F^{\prime}(R)$, then $\cup_{i} A_{i} \in \mathcal{F}(R)$.

Proof. If $A=\cup_{i} A_{i}$ then $A^{l}=\bigcap_{i} A_{i}^{l}$. If $A \cap A^{l} \neq 0$, there would exist elements $A_{1}, \cdots, A_{n}$ in $\left\{A_{i}\right\}$ such that, if $B=A_{1} \cup \ldots \cup A_{n}, B \cap B^{l} \neq 0$. Since each $A_{i}^{l} \in \mathcal{F}(R), B^{l} \in \mathcal{F}(R)$ and therefore $B B^{l} \neq 0$. However, this is contrary to the fact that $A_{i} A_{i}^{l}=0$ for each $i$. This contradiction proves 1.3.

The set $\mathcal{F}^{\prime \prime}(R)$ can be made into a lattice by defining the union operation $\checkmark$ as follows:

$$
A \vee B=(A+B)^{l l}, \quad A, B \in \mathcal{F}^{\prime \prime}(R)
$$

Since $(A+B)^{l l}=\left(A^{l} \cap B^{l}\right)^{l}, A \bigvee B \in \mathcal{F}^{\prime \prime}(R)$ by 1.2 . Clearly $A \bigvee B$ is the least element of $\mathcal{F}^{\prime \prime}(R)$ containing both $A$ and $B$. The mapping $l: A \rightarrow A^{l}$ is a dual automorphism of $\mathcal{F}^{\prime \prime}(R)$, since

$$
(A \vee B)^{l}=A^{l} \cap B^{l}, \quad(A \cap B)^{l}=A^{l} \bigvee B^{l} ; \quad A, B \in F^{\prime \prime}(R)
$$

The unique complement of $A$ in $\mathcal{F}^{\prime \prime}(R)$ is $A^{l}$. Thus the following theorem is a consequence of $[2$, Theorem 17, p. 171].

1.4. TheOREM. The lattice $\mathfrak{F}^{\prime \prime}(R)$ is a Boolean algebra.

For subsets $A$ and $B$ of $R$, let $A B^{-1}=\{r ; r \in R, r B \subset A\}$, and similarly for $B^{-1} A$. Thus $A B^{-1}\left(B^{-1} A\right)$ is the largest subset of $R$ such that $\left(A B^{-1}\right) B C A$ $\left(B\left(B^{-1} A\right) \subset A\right)$. If $A \in \mathfrak{L}(R), B^{-1} A \in \mathfrak{L}(R)$; if $A \in \mathfrak{L}(R)$ and $B \in \mathcal{L}^{\prime}(R)$, then $A B^{-1} \in \mathcal{L}(R)$.

Each $S \in \mathcal{L}^{\prime}(R)$ defines a mapping

$$
\phi_{S}: \phi_{S} A=A \cap S, \quad A \in \mathcal{L}(R),
$$

of $\mathscr{L}(R)$ into $\mathscr{L}(S)$. Clearly $\phi_{S} R=S, \phi_{S}$ is an $\cap$-homomorphism, and $\phi_{S}\left(\cup_{i} A_{i}\right)$ $=\bigcup_{i} \phi_{S} A_{i}$ for each chain $\left\{A_{i}\right\} \subset \mathcal{L}(R)$. Thus $\phi_{S}$ is an $\cap^{\prime}$-map (FI, $\left.\$ 4\right)$ of $\mathfrak{L}(R)$ into $\mathscr{L}(S)$.

Another mapping associated with $S \in \mathscr{L}^{\mathfrak{}}(R)$ is

$$
\theta_{S}: \theta_{S} B=B S^{-1}, \quad B \in \mathcal{L}(S) .
$$

Since $\left(B S^{-1}\right) R S \subset\left(B S^{-1}\right) S \subset B$, evidently $B S^{-1} \in \mathcal{L}(R)$. Also, $S S^{-1}=R$ and $\left(\bigcap_{i} B_{i}\right) S^{-1}=\bigcap_{i} B_{i} S^{-1}$ for every $\left\{B_{i}\right\} \subset \mathcal{L}(S)$. Thus $\theta_{S}$ is an $\bigcap$-map of $\mathscr{L}(S)$ into $\mathscr{L}(R)$.

Associated with each subset $\mathfrak{A}$ of $\mathfrak{L}(R)\left(\mathfrak{B}\right.$ of $\mathfrak{L}(S)$ ) is a subset $\phi_{S} \mathfrak{A}$ of 
$\mathfrak{L}(S)\left(\theta_{S} \mathfrak{B}\right.$ of $\left.\mathfrak{L}(R)\right)$. If $\mathfrak{A}(\mathfrak{B})$ is an inset (FI, $\left.\S 2\right)$, then so is $\phi_{S} \mathfrak{A}\left(\theta_{S} \mathfrak{B}\right)$.

If $\mathfrak{A}$ is a partially ordered set, we shall use the notation $\mathfrak{A} / S=\{A ; A \in \mathfrak{A}$, $A \supset S\}$.

1.5. TheOREM. If $S \in \mathcal{F}^{\prime}(R), \phi_{S}$ is a homomorphism of the inset $\mathfrak{L}^{p}(R)$ onto $\mathfrak{L}^{p}(S)$. Actually, $\phi_{S}$ is an isomorphism of $\mathfrak{L}^{p}(R) / S^{l}$ onto $\mathcal{L}^{p}(S)$ with inverse $\theta_{S}$.

Proof. If $A \in \mathcal{L}^{p}(R)$ and $c P \subset \phi_{S} A$ for some $c \in S$ and $P \in J(S)$, then $c\left(S P R+S^{l}\right) \subset A$. Since $S P R+S^{l} \in \mathfrak{J}(R), c \in A \cap S$ and therefore $\phi_{S} A \in \mathfrak{L}^{p}(S)$.

On the other hand, if $B \in \mathcal{L}^{p}(S)$ and $c P \subset \theta_{S} B$ for some $c \in R$ and $P \in J(R)$, then $c S P S \subset B$ and, since $P S \in J(S), c S \subset B$. Thus $c \in B S^{-1}$ and evidently $\theta_{S} B \in \mathscr{L}^{p}(R)$. It is clear that $\theta_{S} B \supset S^{l}$ and that $\theta_{S} B \cap S=B$ for each $B \in \mathfrak{L}^{p}(S)$. Hence $\phi_{S} \theta_{S}$ is the identity mapping on $\mathscr{L}^{p}(S)$, and $\phi_{S}$ is a homomorphism of $\mathfrak{L}^{p}(R)$ onto $\mathfrak{L}^{p}(S)$.

If $A \in \mathscr{L}^{p}(R) / S^{l}$, then $\theta_{S} \phi_{S} A=(A \cap S) S^{-1} \supset A$. Since $\left(\theta_{S} \phi_{S} A\right) S \subset A \cap S$, $\left(\theta_{S} \phi_{S} A\right)\left(S+S^{l}\right) \subset A$ and, since $S+S^{l} \in J(R), \theta_{S} \phi_{S} A \subset A$. Thus $\theta_{S} \phi_{S}$ is the identity mapping on $\mathfrak{L}^{p}(R) / S^{l}$, and 1.5 follows.

In case $S \in \mathfrak{J}(R), \phi_{S}$ is an isomorphism of $\mathfrak{L}^{p}(R)$ onto $\mathfrak{L}^{p}(S)$ with inverse $\theta_{S}$ according to this theorem.

We mentioned above that $\phi_{S}$ maps insets of $\mathscr{L}(R)$ onto insets of $\mathscr{L}(S)$. Thus $\phi_{S}$ defines a mapping of the set $I(\mathscr{L}(R))$ of all insets of $\mathscr{L}(R)$ into the set $I(\mathscr{L}(S))$ of all insets of $\mathscr{L}(S)$. Similar remarks may be made for $\theta_{S}$.

Since $\phi_{S}$ defines a mapping of $I(\mathscr{L}(R))$ into $I(\mathscr{L}(S))$, it also defines a mapping of the set $C(\mathscr{L}(R))$ of all closure operations on $\mathscr{L}(R)$ into the set $C(\mathscr{L}(S)$ ) of all closure operations on $\mathscr{L}(S)$ in a natural way: for $a \in C(\mathscr{L}(R)), b \in C(\mathscr{L}(S))$,

$$
\phi_{S} a=b \text { if and only if } \phi_{S} \mathscr{L}^{a}(R)=\mathscr{L}^{b}(S) .
$$

If $A \in \mathfrak{L}(S)$, then $A$ is not necessarily in $\mathscr{L}(R)$. However, we may still define $A^{a}$ to be the least element of $\mathscr{L}^{a}(R)$ containing $A$. If $a \in C(\mathscr{L}(R))$ and $b=\phi_{S} a$, then clearly

$$
A^{b}=A^{a} \cap S \quad \text { for every } A \in \mathfrak{L}(S) .
$$

In a similar way, $\theta_{S}$ defines a mapping of $C(\mathscr{L}(S))$ into $C(\mathscr{L}(R))$.

If $S \in F^{\prime}(R)$ and $a \in C(\mathscr{L}(R)) / p$, then $\phi_{S} a \in C(\mathscr{L}(S)) / p$ in view of 1.5 , and similarly for $\theta_{S}$. Thus, if $a \geqq p$ and $b=\phi_{S} a$, then $b \geqq p$ and therefore $A^{p b}=A^{b}$ for every $A \in \mathfrak{L}(S)$. Hence

$$
A^{b}=A^{p a} \cap S \quad \text { for every } A \in \mathfrak{L}(S) .
$$

The advantage of this formula for $A^{b}$ over the previous one is that $A^{p} \in \mathcal{L}(R)$ for every $A \in \mathfrak{L}(S)$.

1.6. THEOREM. If $S \in \mathcal{F}^{\prime}(R), \phi_{S}$ is an $\cap$-homomorphism of $C(\mathscr{L}(R)) / p$ onto $C(\&(S)) / p$, whereas $\theta_{S}$ is a homomorphism of $C(\mathscr{L}(S)) / p$ into $C(\mathscr{L}(R)) / p$. The mapping $\phi_{S}$ carries $C_{m}(\mathcal{L}(R)) / p$ onto $C_{m}(\mathcal{L}(S)) / p$. $I_{j}^{\hat{N}} S \in J(R), \phi_{S}$ is an isomorphism of $C(\mathscr{L}(R)) / p$ onto $C(\mathscr{L}(S)) / p$ with inverse $\theta_{S}$. 
Proof. For $a \in C(\mathscr{L}(R)) / p, \phi_{S} a=b$ where $\phi_{S} \mathscr{L}^{a}(R)=\mathfrak{L}^{b}(S) \subset \mathfrak{L}^{p}(S)$. Evidently $\phi_{S} \theta_{S} \mathcal{L}^{b}=\mathscr{L}^{b}$ by 1.5 . Since $\phi_{S}\left(\bigcup_{i} \mathscr{L}^{a_{i}}\right)=\bigcup_{i} \phi_{S} \mathscr{L}^{a_{i}}$ for every set $\left\{a_{i}\right\}$ $C C(\mathscr{L}(R)) / p, \phi_{S}$ is an $\cap$-homomorphism of $C(\mathscr{L}(R)) / p$ onto $C(\mathscr{L}(S)) / p$ by (FI, 2.2). Clearly $\theta_{S}$ is a homomorphism of $C(\mathscr{L}(S)) / p$ into $C(\&(R)) / p$.

If $a \in C_{m}(\mathcal{L}(R)) / p$ and $b=\phi_{S} a$, then $(A \cap B)^{b}=(A \cap B)^{p a} \cap S=\left(A^{p} \cap B^{p}\right)^{a}$ $\cap S=A^{p a} \cap B^{p a} \cap S=A^{b} \cap B^{b}$ for every $A, B \in \mathscr{L}(S)$, and consequently $b \in C_{m}(\mathscr{L}(S)) / p$. Next, let us assume that $b \in C_{m}(\mathscr{L}(S)) / p$, and let us prove that $a=\theta_{S} b$ is in $C_{m}(\mathscr{L}(R)) / p$. Since $\phi_{S} a=b$, this will prove that $\phi_{S}$ carries $C_{m}(\mathscr{L}(R)) / p$ onto $C_{m}(\mathcal{L}(S)) / p$. Let $C$ be a maximal element of $(\mathscr{L}(R) ; A, B)$ (FI, §2) where $A \in \mathscr{L}(R)$ and $B \in \mathcal{L}^{a}(R)$. Then $C \cap A \subset B$ and $\phi_{S} C \cap \phi_{S} A$ $C \phi_{S} B$. Since $\phi_{S} B \in \mathscr{L}^{b}$ and $\mathscr{L}^{b}$ is an $m$-inset of $\mathscr{L}(S)$, there exists some $D \in \mathscr{L}^{b}$ such that $D \supset \phi_{S} C$ and $D \cap \phi_{S} A \subset \phi_{S} B$. Hence $\theta_{S} D \cap \theta_{S} \phi_{S} A \subset \theta_{S} \phi_{S} B=B$ and, since $\theta_{S} \phi_{S} A \supset A, \theta_{S} D \cap A \subset B$. However, $\theta_{S} D \supset C$, and therefore $\theta_{S} D=C$ due to the maximality of $C$ in $(\mathscr{L}(R) ; A, B)$. Thus $C \in \mathscr{L}^{a}$, and $\mathscr{L}^{a}$ is an $m$-inset of $\mathscr{L}(R)$ by (FI, 2.3). Consequently $a \in C_{m}(\mathscr{L}(R)) / p$.

If $S \in J(R), \phi_{S} a=b$ if and only if $b=\theta_{S} a$, and the last part of the theorem follows readily. This completes the proof of 1.6.

1.7. Theorem. If $S \in \mathcal{F}^{\prime}(R), \phi_{S}$ is a ho, omorphism of $\mathcal{F}^{\prime \prime}(R)$ onto $\mathcal{F}^{\prime \prime}(S)$. Furthermore, $\phi_{S}$ is an isomorphism of $\mathcal{F}^{\prime \prime}(R) / S^{l}$ onto $\mathcal{F}^{\prime \prime}(S)$ with inverse $\theta_{S}$.

Proof. If $A \in \mathcal{F}^{\prime \prime}(R)$, then $\phi_{S} A \in \mathcal{F}^{\prime \prime}(S)$. If $B \in \mathcal{F}^{\prime \prime}(S)$, then $B \in \mathscr{L}^{p}(S)$ so that $B \in \mathfrak{L}(R)$. Actually, $B \in \mathscr{L}^{\prime}(R)$, since $R B B^{l}=0$ and $R B \subset B$. Let $A=\theta_{S} B$ $=B S^{-1}$. Then $A S \subset A \cap S=B$. Now $(A \cap S)^{l} \cap S=A^{l} \cap S=B^{l} \cap S$ and $\left[(A \cap S)^{l} \cap S\right]^{l} \cap S=\left(A^{l} \cap S\right)^{l} \cap S=A^{l l} \cap S=B^{l l} \cap S=B$. Thus $A^{l l} S \subset B$ and $A^{\prime \prime} \subset A$. This proves that $A \in \mathcal{F}^{\prime \prime}(R)$. The rest of the theorem follows from 1.5.

The symbols $\sum$ and $\sum^{*}$ are used to designate the discrete and the full direct sum respectively of the rings of a given set. It may be shown that if $\left\{R_{i}\right\}$ is a set of faithful rings and if $R=\sum_{i} R_{i}$, then

$$
\mathscr{L}^{p}(R)=\left\{\sum_{i} A_{i} ; A_{i} \in \mathscr{L}^{p}\left(R_{\mathbf{i}}\right)\right\}, \quad \mathscr{F}^{\prime \prime}(R)=\left\{\sum_{i} A_{i} ; A_{i} \in \mathcal{F}^{\prime \prime}\left(R_{i}\right)\right\} .
$$

The concept of the universal extension of a faithful ring was introduced in [9]. Since we shall have occasion to use this extension ring frequently, we will sketch its construction.

For the (left) faithful ring $R$, let $\mathfrak{e}\left(R^{+}, R\right)$ be the centralizer of the additive group $R^{+}$of $R$ (considered as a right $R$-module) over the ring $R$. Then we may consider $R^{+}$as a (e, $R$ )-module, in which case $R$ may be considered a subring of $\mathfrak{e}$ due to the faithfulness of $R$. Let the normalizer of $R$ in $\mathfrak{C}$, that is, the largest subring of $C$ containing $R$ as an ideal, be designated by $\bar{R}$. Since $\bar{R}$ has a unit element, it is faithful, and since $R^{l}=0$ in $\mathrm{e}, R \in J(\bar{R})$.

If $S$ is a faithful ring and $R \in J(S)$, and if the mapping $a^{*}$ of $R^{+}$is defined by $a^{*}: a^{*} x=a x, x \in R^{+}, a \in S$, then $S^{*}=\left\{a^{*} ; a \in S\right\} \subset \mathfrak{e}\left(R^{+}, R\right)$. Since $R$ is an ideal in $S, S^{*} \subset \bar{R}$. Thus, up to an isomorphism, $\bar{R}$ is the universal faithful 
ring containing $R$ as an ideal such that $R^{l}=0$ in $\bar{R}$. If $R$ has a unit element, then $\bar{R}=R$; thus, if $S=\bar{R}, \bar{S}=\bar{R}$ for every ring $R$.

1.8. THEOREM. If $\left\{R_{i}\right\}$ is a set of faithful rings and $R=\sum_{i} R_{i}$, then $\bar{R}=\sum_{i}^{*} \bar{R}_{i}$.

Proof (See $[10,4.4])$. Clearly $R \in J\left(\sum_{i}^{*} \bar{R}_{i}\right)$, and therefore $\bar{R} \supset \sum_{i}^{*} \bar{R}_{i}$. Since $R_{j}\left(R_{i} \bar{R}\right)=\left(R_{j} R_{i}\right) \bar{R}=0$ if $i \neq j, \quad R_{i} \bar{R} \subset R_{i}$. Also, $R_{j}\left(\bar{R} R_{i}\right)=\left(R_{j} \bar{R}\right) R_{i}$ $\subset R_{j} R_{i}=0$ if $i \neq j$, and hence $\bar{R} R_{i} \subset R_{i}$. Thus each $R_{i}$ is an ideal of $\bar{R}$. Now for each $r \in \bar{R}, r R_{i} \subset R_{i}$ and $r$ has the same effect on $R_{i}$ as some $r_{i} \in \bar{R}_{i}$. Evidently $r=\sum_{i}^{*} r_{i}$ and $\bar{R} \subset \sum_{i}^{*} \bar{R}_{i}$. This proves 1.8 .

2. Restricted rings. A semi-prime ring is a ring having no nonzero nilpotent ideals [10]. If $R$ is a semi-prime ring, then $\mathscr{L}^{\prime}(R)=\mathscr{F}(R)=\mathcal{F}^{\prime}(R)$, and clearly $\mathcal{F}^{\prime \prime}(R)$ is a complete lattice. This leads us to define a restricted ring as a faithful ring $R$ satisfying the following condition:

$$
\text { If }\left\{A_{i}\right\} \subset \mathcal{F}^{\prime \prime}(R) \text {, then } \bigcap_{i} A_{i} \in \mathscr{F}^{\prime \prime}(R) \text {. }
$$

No example is known to us of a faithful ring that is not restricted.

If it is known that $\bigcap_{i} A_{i} \in \mathcal{F}(R)$, where $\left\{A_{i}\right\} \subset \mathcal{F}^{\prime \prime}(R)$, then it may easily be proved that $\bigcap_{i} A_{i} \in \mathcal{F}^{\prime \prime}(R)$. Thus the definition of a restricted ring is not weakened if the conclusion is just that $\bigcap_{i} A_{i} \in \mathcal{F}(R)$. Defining the union operation $\mathrm{V}$ on $\mathcal{F}^{\prime \prime}(R)$ as previously, $\mathrm{V}_{i} A_{i}=\left(\bigcup_{i} A_{i}\right)^{l l}$, it is evident that $\mathcal{F}^{\prime \prime}(R)$ is a complete Boolean algebra for a restricted ring.

Unless otherwise stated, each ring $R$ considered in this section is assumed to be restricted.

\subsection{TheOREM. Each ideal $S \in \mathcal{F}^{\prime}(R)$ is a restricted ring.}

Proof. If $\left\{A_{i}\right\} \subset \mathcal{F}^{\prime \prime}(S)$, then each $A_{i}=B_{i} \cap S$ for some $B_{i} \in \mathcal{F}^{\prime \prime}(R)$ according to 1.7. Since $\bigcap_{i} A_{i}=\left(\bigcap_{i} B_{i}\right) \cap S$ and $\bigcap_{i} B_{i} \in \mathcal{F}^{\prime \prime}(R), \bigcap_{i} A_{i} \in \mathcal{F}^{\prime \prime}(S)$. This proves 2.1 .

2.2. ThEOREM. If $S$ is a restricted ring and $S \in J(R)$, then $R$ also is restricted.

Proof. If $\left\{A_{i}\right\} \subset \mathcal{F}^{\prime \prime}(R)$ and $A=\bigcap_{i} A_{i}$, then $A \cap S=\bigcap_{i}\left(A_{i} \cap S\right)$ is in $\mathcal{F}^{\prime \prime}(S)$ by assumption. Thus $(A \cap S)^{l} \cap(A \cap S)=0$ and, by 1.1, $A^{l} \cap A \cap S=0$. Hence $A^{l} \cap A=0$ and $A \in \mathcal{F}(R)$. This proves 2.2.

2.3. THEOREM. If $\left\{S_{i}\right\}$ is a set of restricted rings and $R=\sum_{i} S_{i}$, then $R$ also is a restricted ring.

Proof. As previously remarked, $\mathscr{F}^{\prime \prime}(R)=\left\{\sum_{i} A_{i} ; A_{i} \in \mathcal{F}^{\prime \prime}\left(S_{i}\right)\right\}$. If $\left\{B_{j}\right\} \subset \mathcal{F}^{\prime \prime}(R)$, then $B_{j}=\sum_{i} B_{j} \cap S_{i}$ and $\bigcap_{j} B_{j}=\sum_{i}\left(\bigcap_{j}\left(B_{j} \cap S_{i}\right)\right)$. Thus $\bigcap_{j} B_{j} \in F^{\prime \prime}(R)$, and 2.3 follows.

The following theorem gives one condition on a faithful ring that insures it to be restricted. 
2.4. THEOREM. If for the faithful ring $R$ we have $\mathcal{F}(R)=\mathcal{F}^{\prime}(R)$, then $R$ is a restricted ring.

Proof. If $\left\{A_{i}\right\} \subset \mathcal{F}^{\prime \prime}(R)$, then $\mathrm{U}_{i} A_{i}^{l} \in \mathcal{F}(R)$, and hence $\mathcal{F}^{\prime}(R)$, by 1.3. Thus $\left(\cup_{i} A_{i}^{l}\right)^{l}=\bigcap_{i} A_{i} \in \mathcal{F}^{\prime \prime}(R)$.

It is not true that $\mathfrak{F}(R)=\mathcal{F}^{\prime}(R)$ for every restricted ring, as the following example shows.

2.5. Example. Let $F$ be a finite field, and the following rings be subrings of the total matrix ring $F_{4}$ :

$$
S_{1}=F e_{21}, \quad S_{2}=F e_{33}+F e_{43}, \quad S=S_{1}+S_{2} .
$$

Also let

$$
\alpha=e_{11}+e_{22}+e_{44}, \quad R=S+F \alpha .
$$

Clearly $S$ is an ideal of $R$ and $R$ is a faithful restricted ring since its ideal lattice is finite. Now $S_{1}=S_{2}$, and therefore $S_{2} \in \mathcal{F}(R)$. However, $S_{2} \notin \mathcal{F}^{\prime}(R)$ since $S_{2}^{00} \supset S_{1}$. We note that $\mathcal{F}^{\prime \prime}(R)=\{0, R\}$.

Since $\mathcal{F}^{\prime \prime}(R)$ is closed under infinite intersections for a restricted ring $R$, $\mathcal{F}^{\prime \prime}(R)$ induces a closure operation $f$ on $\mathscr{L}(R)$ and $\mathscr{L}^{\prime}(R)$. Thus, for $A \in \mathfrak{L}(R)$, $A^{f}$ is the least element of $\mathcal{F}^{\prime \prime}(R)$ containing $A$. Clearly $f \in C^{0}\left(\mathscr{L}^{\mathfrak{l}}\right)$, although $f$ is not generally in $C_{m}^{0}\left(\mathscr{L}^{\natural}\right)$ as is shown by 2.5 .

\subsection{Lemma. If $a \in C_{m}^{0}(\mathfrak{L})$, then $a \leqq f$.}

Proof. For every $A \in \mathfrak{L}, A^{f} \cap A^{f l}=0$; hence $A \cap A^{f l}=0$ and $A^{a} \cap A^{f l}=0$. Thus $A^{a} A^{f l}=0$ and $A^{a} \subset A^{f}$. This proves 2.6.

2.7. Theorem. If $a \in C_{m}^{0}(\mathfrak{L})$ is atomic (homogeneous) then so is $f$ and $A^{f}$ is an atom of $\mathfrak{L}^{f}$ for every atom $A$ of $\mathfrak{L}^{a}$.

Proof. Let $A$ be an atom of $\mathscr{L}^{a}$. If $0 \neq B \subset A^{f}$ for some $B \in \mathscr{L}^{f}$, then $B \in \mathscr{L}^{a}$ by 2.6. If $A \cap B \neq 0$, then $A \subset B$ due to the atomicity of $A$, and $B=A^{f}$. If $A \cap B=0, A \subset B^{l}$ and therefore $A \subset B^{l} \cap A^{f} \neq A^{f}$ in contradiction to the assumption that $A^{f}$ is the least element of $\mathscr{L}^{f}$ containing $A$. Thus, always, $A^{f}=B$ and $A^{f}$ must be an atom of $\mathscr{L}^{f}$. The rest of the theorem is obvious.

If $a$ is an atomic closure operation on $\mathscr{L}(R)$, let us define

$$
\mathfrak{B}_{a}=\left\{A ; A \text { an atom of } \mathscr{L}^{a}\right\} .
$$

The base $B_{a}$ of $R$ relative to $a$ is defined to be the ring union of all the elements of $\mathfrak{B}_{a}[10, \S 4]$. If $f$ is atomic, then $B_{f}^{l} \in F^{\prime \prime}(R)$ and therefore $B_{f} \in \mathcal{F}^{\prime}(R)$.

2.8. TheOREM. If $f$ is atomic, the base $B_{f}$ of $R$ relative to $f$ is the discrete direct sum of the atoms of $\mathcal{L}^{\prime}(R)$.

Proof. If $\left\{A_{i}\right\} \subset \mathscr{B}_{f}$ and $S=\cup_{i} A_{i}$, then $S^{l}=\bigcap_{i} A_{i}^{l}$, an element of $F^{\prime \prime}(R)$. If $A \cap S \neq 0$ for some $A \in \mathfrak{B}_{f}$, then $A S \neq 0$ and $A A_{i} \neq 0$ for some $i$. Hence 
$A \cap A_{i} \neq 0$, and $A=A_{i}$ since both are atoms of $\mathscr{L}^{f}$. Consequently $S=\sum_{i} A_{i}$ and 2.8 is proved.

If $f$ is atomic and $S$ is an atom of $\mathscr{L}^{\prime}(R)\left(=\mathscr{F}^{\prime \prime}(R)\right)$, then $\mathscr{F}^{\prime \prime}(S)=\{0, S\}$ according to 1.8. Let us call a faithful ring irreducible if $\mathcal{F}^{\prime \prime}(R)=\{0, R\}$. Thus the atoms of $\mathscr{L}^{f}(R)$ are irreducible rings.

We are now in a position to prove the main structure theorems for a restricted ring $R$ having an atomic $m$-closure operation on its lattice of right ideals. We recall from the first section that associated with each faithful ring $S$ is its universal extension ring $\bar{S}$. The following theorem is analogous to $[10,4.5]$.

\subsection{Theorem. If $f$ is atomic and $C=B_{f}^{l}$, then $B_{f} \oplus C \subset R \subset \bar{B}_{f}+\bar{C}$.}

Proof. Since $B_{f}+C_{f}^{l} \in J(R)$, the completion, $\bar{B}_{f}+\bar{C}_{f}^{l}$, contains $R$. This proves 2.9.

The restricted ring $B_{f}^{l}$ appearing in the theorem above can have no atomic $m$-closure operation on its lattice of right ideals, since clearly $f$ is nonatomic on $B_{f}^{l}$.

If $f$ is homogeneous, then $B_{f}^{l}=0$ and we have the following corollary of 2.9 and 1.8 .

2.10. Corollary. If $f$ is homogeneous, there exist irreducible rings $A_{i}$ such that

$$
\sum_{i} A_{i} \subset R \subset \sum_{i}^{*} \bar{A}_{i}
$$

The converse of 2.10 which is analogous to $[10,4.6]$ is the following result.

2.11. ThEOREM. If $\left\{A_{i}\right\}$ is a set of irreducible rings and if $R$ is a ring such that

$$
\sum_{i} A_{i} \subset R \subset \sum_{i}^{*} \bar{A}_{i}
$$

then $R$ is a restricted ring and $f$ is a homogeneous closure operation on $\mathscr{L}^{\prime}(R)$ for which $\mathfrak{B}_{f}=\left\{\bar{A}_{i} \cap R\right\}$.

Proof. Each $A_{i}$ is a restricted ring, and hence $S=\sum_{i} A_{i}$ is a restricted ring by 2.3. Since $R \subset \bar{S}, S \in J(R)$ and $R$ is a restricted ring according to 2.2. By $1.6, \theta_{S}$ is an isomorphism of $\mathcal{F}^{\prime \prime}(S)$ onto $\mathcal{F}^{\prime \prime}(R)$. Since $f$ is homogeneous on $\mathcal{L}^{\prime}(S), f$ must be homogeneous on $L^{\prime}(R)$. The atoms of $\mathcal{F}^{\prime \prime}(R)$ have the form $\theta_{S} A_{i}=A_{i} S^{-1}=\bar{A}_{i} \cap R$. This proves 2.11 .

3. Reducible rings. A completely reducible ring is a ring $R$ such that $\mathcal{L}^{\mathfrak{}}(R)$ is a complemented modular lattice. It is well-known (see, for example, $[3$, Theorem 2]) that a completely reducible ring is a discrete direct sum of simple rings.

As a generalization of complete reducibility, let us call a faithful ring $R$ reducible if for every pair $A, B$ of ideals of $R$ for which $A \cap B=0$, there exist ideals $A^{\prime} \supset A$ and $B^{\prime} \supset B$ such that $A^{\prime} \cap B^{\prime}=0$ and $A^{\prime}+B^{\prime} \in J(R)$. A semi- 
prime ring is reducible since $A \cap A^{l}=0$ and $A+A^{l} \in J(R)$ for every ideal $A$ of $R[10, \S 1]$. We give now another example of a reducible ring.

3.1. Example. Let $F$ be a field and the rings described below be subrings of $F_{4}$.

Also let

$$
A=F e_{11}+F e_{21}, \quad B=F e_{33}+F e_{43}, \quad S=A+B .
$$

$$
\alpha=e_{22}+e_{44}, \quad R=S+F \alpha .
$$

Since $R$ has a unit element, $R$ is faithful. Evidently $S \in J(R)$, and $S^{r}=C+D$ $+F \alpha$ where

$$
C=F e_{21}, \quad D=F e_{43} .
$$

The ideal lattice of $R$ may be verified to be as in the figure. Since $A+B \in J(R)$,

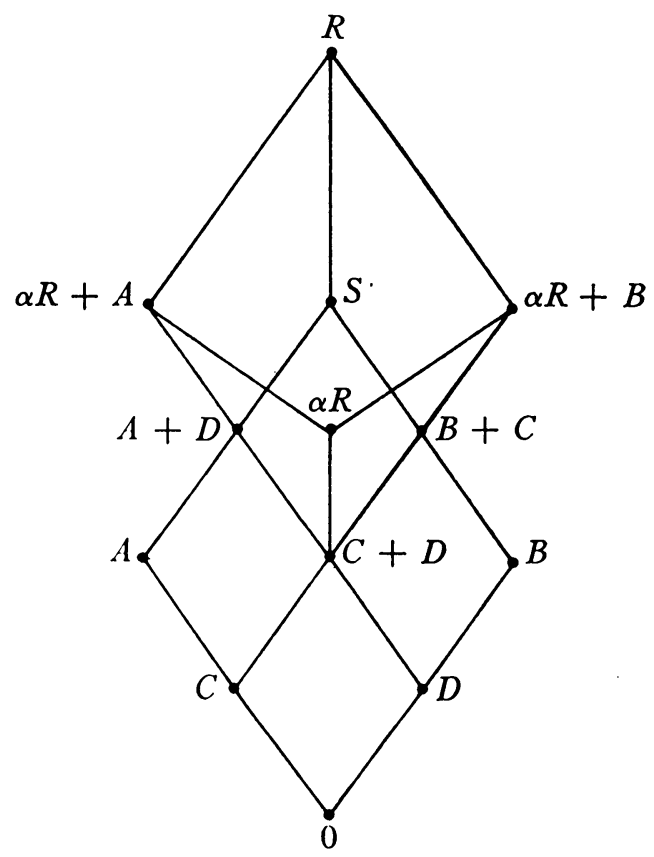

$$
\mathfrak{L}^{\mathfrak{}}(R)
$$

it is evident from this figure that $R$ is a reducible ring. Also, $\mathcal{F}^{\prime \prime}(R)=\{0, A, B$, $R\}$. An interesting feature of this example is that $R$ is not reducible as a right faithful ring, since $S^{r} \neq 0$.

If $H=F e_{11}+F e_{21} \subset F_{2}$, then $H$ is isomorphic to rings $A$ and $B$. The centralizer of $H$, considered as a right $H$-module, is just $F_{2}$. It is clear that the universal extension ring $\bar{H}$ is given by

$$
\bar{H}=F e_{11}+F e_{21}+F e_{22} \text {. }
$$

The structure of $R$ in 2.10 has the form

$$
A \oplus B \subset R \subset \bar{A} \oplus \bar{B},
$$


where $\bar{A}$ and $\bar{B}$ are isomorphic to $\bar{H}$.

3.2. THEOREM. If $R$ is reducible, then $\mathfrak{F}(R)=\mathscr{F}^{\prime}(R)$.

Proof. Let $A \in \mathcal{F}(R)$, so that $A \cap A^{l}=0$. Since $R$ is reducible, there exist ideals $B \supset A$ and $B^{\prime} \supset A^{l}$ such that $B \cap B^{\prime}=0$ and $B+B^{\prime} \in J(R)$. Now $B^{\prime} \subset B^{l}$ and $B^{l} \subset A^{l}$, and therefore $A^{l}=B^{\prime}$. Since $B \cap A^{l}=0, A^{l} \subset B^{l}$ and, in view of our previous remarks, $A^{l}=B^{l}$. Thus $\left(B+A^{l}\right)^{l}=B^{l} \cap A^{l l}=0, A^{l} \cap A^{l l}=0$, and $A \in \mathcal{F}^{\prime}(R)$. This proves 3.2.

If $R$ is reducible, $R$ is restricted in view of 3.2 and 2.4. Thus $\mathcal{F}^{\prime \prime}(R)=\mathscr{L}^{f}(R)$, where $f \in C^{0}\left(\mathscr{L}^{\prime}(R)\right)$. We shall now determine the nature of the closure operation $f$.

We recall that if $A, B \in \mathcal{L}^{\prime}(R), B$ is called an essential extension (FI, §6) of $A$ in $\mathscr{L}^{\prime}(R)$ if $A \subset B$ and for every $C \in \mathcal{L}^{\prime}(R)$ such that $C \cap B \neq 0$ also $C \cap A \neq 0$. The set $\mathcal{E}(A)$ of all essential extensions of $A$ has maximal elements.

3.3. Theorem. For every ideal $A$ of the reducible ring $R, A^{f}$ is the unique maximal essential extension of $A$ in $\mathfrak{L}^{\prime}(R)$.

Proof. Let $B$ be a maximal element of $\left(\mathcal{L}^{\imath} ; A, 0\right)$ and $C$ be a maximal ele-

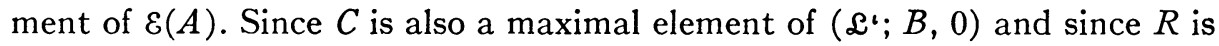
reducible, $C+B \in J(R), B=C^{l}$, and $C=B^{l}$. Thus $C \in \mathcal{F}^{\prime \prime}(R)$ and $A^{f} C C$ since $A^{f}$ is the least element of $\mathcal{F}^{\prime \prime}(R)$ containing $A$. On the other hand, $A^{f} \in \mathcal{F}^{\prime \prime}(R)$ and $A^{f l} \cap A^{f}=0$. Since $A \cap A^{f l}=0$, necessarily $C \cap A^{f l}=0$ and $C \subset A^{f l l}=A^{f}$. Thus $C=A^{f}$, and the theorem is proved.

3.4. THEOREM. If $R$ is a reducible ring, the closure operation $f$ is the maximal element of $C_{m}^{0}\left(\mathscr{L}^{\mathfrak{d}}\right)$.

Proof. Let us first prove that $f \in C_{m}^{0}\left(\mathscr{L}^{\natural}\right)$. To this end, let $A \in \mathcal{L}^{\natural}, B \in \mathcal{L}^{f}$, and $C$ be a maximal element of $\left(\mathcal{L}^{\prime} ; A, B\right)$. If $D \supset C$, then $D \cap A \nsubseteq B$ and $E=D \cap A \cap B^{l} \neq 0$. Since $E \cap D \neq 0$ whereas $E \cap C \subset B \cap B^{l}=0, D \notin \varepsilon(C)$. Hence $\mathcal{E}(C)=\{C\}$, and $C=C^{f}$ by 3.3. Thus $f \in C_{m}^{0}\left(\mathscr{L}^{\imath}\right)$ by (FI, 2.3).

The closure operation $f$ is reducible in the sense of (FI, §6). Hence, by (FI, 6.3), $f$ is the $\cap$-identity element of $C_{m}^{0}\left(\mathcal{L}^{\mathfrak{l}}\right)$. This completes the proof of 3.4 .

3.5. TheOREM. If $\left\{S_{i}\right\}$ is a set of reducible rings and $R=\sum_{i} S_{i}$, then $R$ also is a reducible ring.

Proof. Let $A$ and $B$ be maximal ideals of $R$ such that $A \cap B=0$. We wish to prove that $A+B \in J(R)$. Since $A, B \in \mathcal{L}^{p}(R), A=\sum_{i} A_{i}$ and $B=\sum_{i} B_{i}$ for some $A_{i}, B_{i} \in \mathcal{L}^{p}\left(S_{i}\right)$. Clearly $A_{i}$ and $B_{i}$ are maximal ideals of $S_{i}$ such that $A_{i} \cap B_{i}=0$. Thus $A_{i}+B_{i} \in J\left(S_{i}\right)$ for each $i$. If $c(A+B)=0$, where $c=\sum_{i} c_{i}$, $c_{i} \in S_{i}$, then $c_{i}\left(A_{i}+B_{i}\right)=0$ and $c_{i}=0$. Thus $c=0, A+B \in J(R)$, and 3.5 is proved. 
3.6. ThEOREM. If $S$ is a reducible ring and $S \in J(R)$, then $R$ also is a reducible ring.

Proof. Let $A$ and $B$ be ideals of $R$ for which $A \cap B=0$, and let $A^{\prime}=A \cap S$ and $B^{\prime}=B \cap S$. By assumption, there exist ideals $C \supset A^{\prime}$ and $D \supset B^{\prime}$ of $S$ such that $C \cap D=0, C^{l} \cap S=D$, and $D^{l} \cap S=C$. Since $C, D \in \mathscr{L}^{p}(S), C, D \in \mathscr{L}(R)$ and $S C, S D \in \mathcal{L}^{\prime}(R)$. If $a(S C+S D)=0$, then $a S(C+D)=0, a S=0$, and, finally, $a=0$. Thus $S C \cap S D=0$ and $S C+S D \in J(R)$. Now $(A+S C) \cap S \subset C$ and $(B+S D) \cap S \subset D$, and therefore $(A+S C) \cap(B+S D) \cap S=0$. Since $S \in J(R),(A+S C) \cap(B+S D)=0$, and $R$ is a reducible ring.

The converse of the preceding theorem does not hold, as is shown by the following example.

3.7. ExAmple. Let $F$ be the held of integers modulo 2, and $S$ and $R$ be the following subrings of $F_{3}$ :

$$
S=F e_{11}+F e_{21}+F e_{31}, \quad R=S+F e_{32} .
$$

Clearly $R$ and $S$ are left faithful rings, and $S \in J(R)$. The ideal lattices of $R$ and $S$ are shown in the figure. We see that trivially $R$ is reducible, whereas $S$ is not reducible since $e_{21} S+e_{31}^{0} S$ is not in $\Im(S)$.
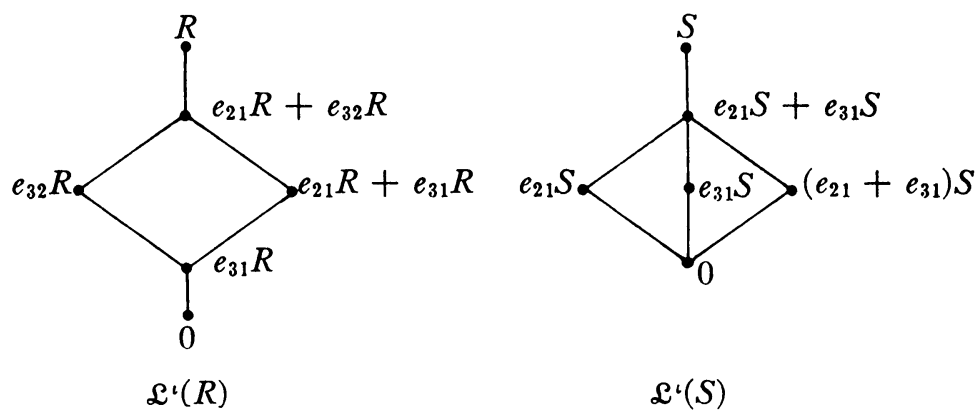

The fact that $R$ and $S$ are one-sided faithful rings in the example above is important. Thus, it can be shown that if $R$ is both left and right faithful and left and right reducible, then each ideal of $R$ that is faithful is reducible.

Let us call a ring $R$ strongly irreducible ( $s$-irreducible) if $A \cap B \neq 0$ for every pair $A, B$ of nonzero ideals of $R$. The ring $R$ of 3.7 is $s$-irreducible. Every $s$-irreducible ring is trivially reducible. It is not known if the atoms of $\mathscr{L}^{f}(R)$ need be $s$-irreducible if $R$ is reducible. However, the following result is true. that

3.8. THEOREM. If $\left\{A_{i}\right\}$ is a set of s-irreducible rings and if $R$ is a ring such

$$
\sum_{i} A_{i} \subset R \subset \sum_{i}^{*} \bar{A}_{i},
$$

then $R$ is a reducible ring and $f$ is a homogeneous closure operation on $\mathscr{L}^{\prime}(R)$ with $\mathfrak{B}_{f}=\left\{\bar{A}_{i} \cap R\right\}$. 
Proof. The ring $\sum_{i} A_{i}$ is reducible by 3.5 , and therefore $R$ is reducible by 3.6. The rest of the theorem follows from 2.11 .

4. Modules of a faithful ring. The ring $R$ encountered in this section is assumed to be faithful. For each $x \in R$ and $A \in \mathcal{L}(R), x^{-1} A \in \mathcal{L}(R)$ and $x^{-1}$ is an $\cap$-endomorphism of $\mathscr{L}(R)$. Since $x^{-1}\left(\cup_{i} A_{i}\right)=U_{i} x^{-1} A_{i}$ for every chain $\left\{A_{i}\right\} \subset \mathscr{L}(R), x^{-1}$ is an $\cap^{\prime}$-map (FI, $\left.\S 4\right)$ of $\mathfrak{L}(R)$ into $\mathfrak{L}(R)$.

A closure operation $a$ on $\mathscr{L}(R)$ commutes with $x^{-1}$ if $a x^{-1}=x^{-1} a$, that is, if $\left(x^{-1} A\right)^{a}=x^{-1} A^{a}$ for every $A \in \mathcal{L}(R)$. Let

$$
C^{\prime}(\mathscr{L})=\left\{a ; a \in C(\mathscr{L}), a x^{-1}=x^{-1} a \text { for every } x \in R\right\} .
$$

By $(\mathrm{FI}, 4.9), C^{\prime}(\mathfrak{L})$ is a complete sublattice of $C(\mathfrak{L})$. That $p \in C^{\prime}(\mathfrak{L})$ follows from $(F I, 5.2)$.

4.1. Lemma. If $s \in C^{\prime}(\mathcal{L}) / p$, then $A^{s}=\left\{r ; r \in R,\left(r^{-1} A\right)^{s}=R\right\}$ for every $A \in \mathcal{L}$.

Proof. If $\left(r^{-1} A\right)^{s}=R$ then $r^{-1} A^{s}=R, r R \subset A^{*}$, and, since $s \geqq p, r \in A^{*}$. Conversely, if $r \in A^{s}$ then $\left(r^{-1} A\right)^{s}=r^{-1} A^{*}=R$.

4.2. Lemma. If $s \in C^{\prime}(\mathfrak{L}) / p$, then $s \in C\left(\mathscr{L}^{\prime}\right)$.

Proof. If $A \in \mathcal{L}^{\prime}(R), r \in R$ and $c \in A^{s}$, then $(r c)^{-1} A=c^{-1}\left(r^{-1} A\right) \supset c^{-1} A$. Hence $\left((r c)^{-1} A\right)^{s}=R$ and $r c \in A^{s}$. Thus $A^{s} \in \mathcal{L}^{\prime}(R)$.

An immediate consequence of this lemma is that the bound of every element of $\mathscr{L}^{s}(R)$ also is in $\mathscr{L}^{s}(R)$.

Let us turn now to a discussion of modules of a faithful ring $R$. Associated with each (right) $R$-module $M$ is its lattice of submodules $\mathfrak{M}(M)$. An $N \in \mathfrak{M}(M)$ is called prime if and only if

$$
x S \subset N, \quad x \in M \text { and } S \in J(R), \text { implies } x \in N .
$$

We shall again designate by $p$ the closure operation on $\mathscr{T}(M)$ induced by the set of all prime submodules of $M$. It is proved in (FI, §5) that $p \in C_{m}(\mathfrak{T})$. The module $M$ itself is called prime if 0 is a prime submodule, that is, if $p \in C_{m}^{0}(\mathfrak{T})$.

For subsets $A$ and $B$ of the $R$-module $M$, let $B^{-1} A=\{r ; r \in R, B r \subset A\}$. If $A \in \mathscr{M}(M)$, then $B^{-1} A \in \mathfrak{L}(R)$; if $A \in \mathscr{N}^{p}, B^{-1} A \in \mathcal{L}^{p}(R)$. This last statement follows from the observation that if $r S \subset B^{-1} A$ for some $r \in R$ and $S \in J(R)$, then $B r S \subset A, B r \subset A$, and finally $r \in B^{-1} A$.

If $s \in C(\mathscr{L})$, an $R$-module $M$ is called $s$-admissible if and only if $x^{-1} 0 \in \mathfrak{L}^{s}(R)$ for every $x \in M$. For example, if $M$ is prime then $M$ is $p$-admissible.

It will be assumed henceforth in this section that $R$ is a ring and $s$ is a closure operation on $\mathscr{L}(R)$ such that

$$
s \in C_{m}^{0}(\mathfrak{L}) / p,
$$

and that $M$ is a prime $s$-admissible module. 
If $N \in \mathscr{M}(M)$ and $x, y \in M$ such that $\left(x^{-1} N\right)^{s}=\left(y^{-1} N\right)^{s}=R$, and if $r \in R$, then $\left[(x-y)^{-1} N\right]^{s} \supset\left[\left(x^{-1} N\right) \cap\left(y^{-1} N\right)\right]^{s}=R$, and $\left[(x r)^{-1} N\right]^{s}=\left[r^{-1}\left(x^{-1} N\right)\right]^{s}$ $=r^{-1}\left(x^{-1} N\right)^{8}=r^{-1} R=R$. Hence, if for each $N \in \Re(M)$ we define

$$
N^{t}=\left\{x ; x \in M,\left(x^{-1} N\right)^{s}=R\right\},
$$

then $N^{\imath} \in \mathfrak{M}(M)$ and $t=t(s)$ is at least a quasi-closure operation on $\mathfrak{T}(M)$.

4.3. Theorem. For every $x \in M, s x^{-1}=x^{-1} t$.

Proof. We must prove that for every $N \in \mathfrak{M}(M),\left(x^{-1} N\right)^{s}=x^{-1} N^{t}$. If $r \in\left(x^{-1} N\right)^{s}$, then $\left[r^{-1}\left(x^{-1} N\right)\right]^{s}=R$ by 4.1 . Hence $\left[(x r)^{-1} N\right]^{*}=R, x r \in N^{t}$, and $r \in x^{-1} N^{t}$. Thus $\left(x^{-1} N\right)^{8} C x^{-1} N^{t}$. A reversal of this argument proves that $x^{-1} N^{t} \subset\left(x^{-1} N\right)^{8}$, and establishes 4.3.

Since $\left(x^{-1} N^{t}\right)^{s}=\left(x^{-1} N\right)^{s s}=\left(x^{-1} N\right)^{s},\left(x^{-1} N^{t}\right)^{s}=R$ if and only if $\left(x^{-1} N\right)^{s}$ $=R$. Consequently $N^{t t}=N^{t}$ for every $N \in \mathscr{T}(M)$, and $t$ is a closure operation on $\mathscr{T}(M)$. Actually $t \in C^{0}(\mathfrak{T})$, since $\left(x^{-1} 0\right)^{\bullet}=x^{-1} 0$ and $x^{-1} 0=R$ if and only if $x=0$.

4.4. Lemma. If $K, N \in \mathfrak{T}^{p}(M)$ and $x^{-1} K=x^{-1} N$ for every $x \in M$, then $K=N$.

Proof. If $x \in K$, then $x^{-1} N=R, x R \subset N$, and $x \in N$. Hence $K \subset N$. Similarly $N \subset K$, and 4.4 follows.

4.5. Theorem. $t \in C^{\prime}{ }_{m}(\mathfrak{T}) / p$; and if $s=p$ then $t=p$ also.

Proof. If $x S \subset N^{t}$ for some $x \in M$ and $S \in J(R)$, then $S \subset\left(x^{-1} N\right)^{8}$ and, since $S^{p}=R$ and $p \leqq s,\left(x^{-1} N\right)^{s}=R$ and $x \in N^{t}$. Hence $N^{t p}=N^{t}$ and $p \leqq t$ by (FI, 1.6).

To prove that $t \in C_{m}(\mathfrak{N})$, let $K, N \in \mathscr{N}(M)$. Then for every $x \in M$, $x^{-1}(K \cap N)^{t}=\left[x^{-1}(K \cap N)\right]^{*}=\left(x^{-1} K \cap x^{-1} N\right)^{*}=\left(x^{-1} K\right)^{s} \cap\left(x^{-1} N\right)^{\text {s }}$ $=x^{-1} K^{t} \cap x^{-1} N^{t}=x^{-1}\left(K^{t} \cap N^{t}\right)$. Hence $(K \cap N)^{t}=K^{t} \cap N^{t}$ by 4.4.

Finally, we know from $(\mathrm{FI}, 5.2)$ that $\left(x^{-1} N\right)^{p}=x^{-1} N^{p}$ for every $x \in M$ and $N \in \mathfrak{M}(M)$. Since $\left(x^{-1} N\right)^{8}=x^{-1} N^{t}$ by $4.3, t=p$ if $s=p$ by 4.4 .

4.6. ThEOREM. If $s$ is atomic and if $x A \neq 0$ for some $x \in M$ and atom $A \in \mathscr{L}^{s}(R)$, then $t$ is atomic and $(x A)^{t}$ is an atom of $\mathfrak{T}^{t}(M)$.

Proof. This follows from (FI, 4.10).

If $N \in \mathscr{M}(M)$ and $K \in \mathscr{M}^{t}(M)$ with $K \subset N$, then the $R$-module $N-K$ is prime and $\mathfrak{T}(N-K)=\{A-K ; A \in \mathfrak{T}(M), K \subset A \subset N\}$. Since $(x+K)^{-1}$ $\cdot(A-K)=x^{-1} A,(x+K)^{-1} 0 \in \mathscr{L}^{s}(R)$ and $N-K$ is $s$-admissible. The closure operation induced on $N-K$ by $s$ is the obvious one, as stated in the following theorem.

4.7. ThEOREM. If $N \in \mathscr{T}(M)$ and $K \in \mathscr{T}^{t}(M)$ with $K \subset N$, then the closure operation $u$ on $\mathfrak{T}(N-K)$ induced by $s$ is given by 


$$
\mathfrak{M}^{u}(N-K)=\left\{(A \cap N)-K ; A \in \mathfrak{M}^{t}(M), A \supset K\right\} .
$$

Proof. If $x \in N$ and $A \in \mathscr{T}^{t}(M)$ with $A \supset K$, then $(x+K)^{-1}[(A \cap N)-K]$ $=x^{-1}(A \cap N)=x^{-1} A=R$ if and only if $x \in A$. Thus $(A \cap N)-K \in \mathbb{T}^{u}(N-K)$. On the other hand, if $B-K \in \mathfrak{T}^{u}(N-K)$, where $K \subset B \subset N$, and $x \in B^{t} \cap N$, then $\left(x^{-1} B\right)^{s}=R$ and $\left[(x+K)^{-1}(B-K)\right]^{s}=R$. Hence $x+K \in B-K$ and $x \in B$. This proves that $B^{t} \cap N=B$, and completes the proof of 4.7.

This theorem allows us to construct examples of the modules considered in this section. We need only select $M=R^{+}, K \in \mathscr{L}^{s}(R)$, and $N \in \mathfrak{L}(R)$ in 4.7 to get a module $N-K$ of the type being considered.

Each of the sets $\mathcal{L}^{s}(R)$ and $\boldsymbol{M}^{t}(M)$ can be made into a lattice by defining the union operation $\bigvee$ in the obvious way. Thus, for $\left\{N_{i}\right\} \subset \mathscr{M}^{t}(M), \bigvee_{i} N_{i}$ $=\left(\bigcup_{i} N_{i}\right)^{t}$. The lattices so formed are modular by $(\mathrm{FI}, 6.1)$.

5. Annihilating submodules. In this section, $M$ is assumed to be a prime $R$-module of a faithful ring $R$. For each subset $A$ of $R$, let ${ }^{l} A$ designate the (left) annihilator of $A$ in $M$.

5.1. Lemma. If $A \in \mathcal{F}^{\prime}(R)$, then ${ }^{l} A=\left(M A^{l}\right)^{p}$.

Proof. We first prove that ${ }^{l} A \in \mathfrak{M}^{p}(M)$. If $x S C^{l} A$ for some $x \in M$ and $S \in J(R)$, then $x S A=0, x A S A=0$, and, finally, $x A S\left(A+A^{l}\right)=0$. Since $A+A^{l} \in J(R), x A S=0$ and $x A=0$ by the primeness of $M$. Hence $x \in{ }^{l} A$ and ${ }^{l} A \in \mathfrak{M}^{p}(M)$.

Clearly $M A^{l} \subset^{l} A$, and therefore $\left(M A^{l}\right)^{p} C^{l} A$. If $x \in \epsilon^{l} A$, then $x\left(A+A^{l}\right)$ $=x A^{l} \subset\left(M A^{l}\right)^{p}$ and $x \in\left(M A^{l}\right)^{p}$. Thus ${ }^{l} A \subset\left(M A^{l}\right)^{p}$, and the lemma is proved.

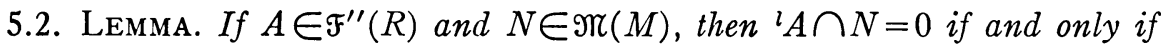
$N \subset^{l}\left(A^{l}\right)$.

Proof. By 5.1, ${ }^{l} A=\left(M A^{l}\right)^{p}$ and ${ }^{l}\left(A^{l}\right)=(M A)^{p}$. Since $\left(M A^{l} \cap M A\right)$ $\cdot\left(A+A^{l}\right)=0, M A^{l} \cap M A=0$; and since $p \in C_{m}^{0}(\mathfrak{T}),{ }^{l} A \cap^{l}\left(A^{l}\right)=0$. Now if ${ }^{\prime} A \cap N=0$ for some $N \in \mathfrak{M}(M)$, then $N A^{l} C^{l} A \cap N=0$ and $N C^{l}\left(A^{l}\right)$. This proves 5.2 .

5.3. Lemma. If $A, B \in \mathcal{F}^{\prime \prime}(R)$, then ${ }^{l}(A \bigvee B)={ }^{l} A \cap^{l} B$.

Proof. Clearly ${ }^{l}(A \bigvee B) \subset^{l} A \cap \cap^{l} B$. If $C=A+B$, then $C^{l}=A^{l} \cap B^{l}=(A \vee B)^{l}$. Now $\left[{ }^{l}\left(C^{l}\right) \cap^{l} A \cap^{l} B\right]\left(C^{l}+C\right)=0$, and therefore (since $\left.C^{l}+C \in J(R)\right)^{l}\left(C^{l}\right)$ $\cap^{l} A \cap^{l} B=0$. Thus ${ }^{l} A \cap^{l} B C^{l}\left(C^{l l}\right)={ }^{l}(A \vee B)$, and the lemma is proved.

The set

$$
\mathfrak{H}(\mathfrak{T T}(M))=\left\{{ }^{\prime} A ; A \in \mathcal{F}^{\prime \prime}(R)\right\}
$$

is closed under the intersection operation by 5.3. It can be made into a lattice by defining ${ }^{l} A \vee^{l} B={ }^{l}(A \cap B),{ }^{l} A,{ }^{l} B \in \mathcal{F}(\mathfrak{N})$. It may be verified that ${ }^{\imath} A \bigvee^{l} B$ actually is the least element of $\mathcal{H C}(\mathfrak{T T})$ containing both ${ }^{\prime} A$ and ${ }^{l} B$.

5.4. TheOREm. The lattice $\mathfrak{H C}(\mathfrak{T})$ is a Boolean algebra. If ${ }^{l} A \neq M$ for every 
nonzero $A \in \mathcal{F}^{\prime \prime}(R)$, then $\mathfrak{F}^{\prime \prime}(R)$ and $\mathfrak{H}(\mathfrak{T})$ are dual isomorphic under the correspondence $A \rightarrow^{l} A$.

Proof. It is evident that ${ }^{l} A \cap^{l}\left(A^{l}\right)=0$ and ${ }^{l} A \bigvee^{l}\left(A^{l}\right)=M$. If ${ }^{l} A \cap \cap^{l} B=0$ and ${ }^{l} A \bigvee^{l} B=M$ for some $A, B \in \mathcal{F}^{\prime \prime}(R)$, then ${ }^{l} B C^{l}\left(A^{l}\right)$. If we let $C=A^{l} \bigvee B$, then ${ }^{l} C={ }^{l} B$ so that ${ }^{l} A \cap{ }^{l} C=0$ and ${ }^{l} A \bigvee^{l} C=M$. Now $C=A^{l} \bigvee(A \cap C)$, and therefore ${ }^{l} C={ }^{l}\left(A^{l}\right) \cap\left({ }^{l} A \bigvee^{l} C\right)={ }^{l}\left(A^{l}\right)$. Hence each ${ }^{l} A \in \mathcal{H C}(\mathfrak{T})$ has a unique complement ${ }^{l}\left(A^{l}\right)$, and $\mathfrak{H C}(\mathfrak{T})$ is a Boolean algebra.

If ${ }^{l} A \neq M$ for every nonzero $A \in \mathcal{F}^{\prime \prime}(R)$ and if ${ }^{l} A={ }^{l} B$ for some $A, B$ $\in \mathcal{F}^{\prime \prime}(R)$, then ${ }^{l}\left(A \cap B^{l}\right)={ }^{l} A \bigvee^{l}\left(B^{l}\right)={ }^{l} B \bigvee^{l}\left(B^{l}\right)=M$ and $A \cap B^{l}=0$. Thus $A \subset B$. Similarly one proves that $B \subset A$, and therefore $A=B$. Hence $\mathcal{F}^{\prime \prime}(R)$ and $\mathscr{H}(\mathfrak{T})$ are dual isomorphic, and 5.4 is proved.

If $M=R^{+}$, then it is clear that ${ }^{~} A=A^{l}$ for each $A \in F^{\prime \prime}(R)$, and the mapping $A \rightarrow^{l} A$ is essentially the dual automorphism of $R$ defined in the Boolean algebra $\mathcal{F}^{\prime \prime}(R)$.

The lattice $\mathfrak{H C}(\mathfrak{T})$ is quite easily shown to be a complete Boolean algebra in case $R$ is a restricted ring. Also, $\mathfrak{H C}(\mathfrak{T})$ is dual isomorphic with $\mathscr{F}^{\prime \prime}(R) / C$ (and also $\mathcal{F}^{\prime \prime}\left(C^{l}\right)$ ) if $R$ is restricted, where $C$ is the maximal annihilator of $M$ in $\mathcal{F}^{\prime \prime}(R)$. In case $R$ is restricted, the lattice $\mathscr{H}(\mathfrak{T})$ induces a closure operation $g$ on $\mathscr{M}(M)$; for $N \in \mathscr{M}(M), N^{o}$ is the least element of $\mathcal{H C}(\mathfrak{N})$ containing $N$.

6. Rings with nonsingular elements. For $A, B \in \mathcal{L}(R)$, we shall write $A C^{\prime} B$ if $B$ is an essential extension of $A$. The elements of $\mathscr{L}(R)$ having $R$ as an essential extension are of particular importance in the following discussion. Thus we shall let

$$
\mathscr{L}^{\Lambda}(R)=\left\{A ; A \in \mathfrak{L}(R), A \subset^{\prime} R\right\} .
$$

If $M$ is an $R$-module, then we shall also let

$$
\mathfrak{m} \wedge(M)=\left\{N ; N \in \mathfrak{M}(M), N \subset^{\prime} M\right\} .
$$

Clearly $\mathfrak{L}^{\mathbf{\Lambda}}$ and $\mathscr{T} \boldsymbol{\Lambda}$ are lattices, although not usually complete lattices.

Contained in $R$ and $M$ are the subsets $R^{\mathbf{\Lambda}}$ and $M^{\boldsymbol{\Lambda}}$ defined by

$$
R^{\mathbf{\Delta}}=\left\{r ; r \in R, r^{-1} 0 \in \mathscr{L}^{\mathbf{\Delta}}(R)\right\}, \quad M^{\mathbf{\Delta}}=\left\{x ; x \in M, x^{-1} 0 \in \mathscr{L}^{\mathbf{\Delta}}(R)\right\} .
$$

It may be shown that $R^{\mathbf{\Delta}}$ is an ideal of $R$ and $M^{\mathbf{\Delta}}$ is a submodule of $M$. We call $R^{\mathbf{\Lambda}}$ the singular ideal [6, p. 894] of $R$ and $M^{\mathbf{\Lambda}}$ the singular submodule of $M$.

If $N \in \mathscr{M}(M)$, evidently $N^{\boldsymbol{\Lambda}}=M^{\boldsymbol{\Delta}} \cap N$. Therefore, if $N C^{\prime} M, N^{\boldsymbol{\Delta}}=0$ if and only if $M^{\mathbf{\Lambda}}=0$. Also, if $R^{\mathbf{\Lambda}} \neq 0$ and $M R^{\mathbf{\Lambda}} \neq 0$, then $M^{\mathbf{\Delta}} \supset M R^{\mathbf{\Lambda}} \neq 0$.

We shall assume henceforth in this section that $R$ and $M$ are so chosen that

$$
R^{\boldsymbol{\Lambda}}=0 \text { and } M^{\boldsymbol{\Delta}}=0 .
$$

Under these restrictions, $A^{l}=0$ and ${ }^{l} A=0$ for every $A \in \mathcal{L}^{\Delta}(R)$.

If $B \in \mathscr{L}(R)$ and $B^{\prime}$ is a complement of $B$, then $B+B^{\prime} \in \mathscr{L}^{\wedge}(R)$ and $\left(B+B^{\prime}\right)^{l}=0$. In particular, if $A \in \mathcal{F}(R)$, then $A^{l}$ is the unique complement of 
$A$ and $\left(A+A^{l}\right)^{l}=A^{l} \cap A^{l l}=0$. Therefore $\mathcal{F}(R)=\mathscr{F}^{\prime}(R)$, and we conclude from 2.4 that $R$ is a restricted ring if $R^{\Delta}=0$.

6.1. Theorem. If $N \in \mathfrak{M}(M), N \subset^{\prime} M$ if and only if $x^{-1} N \in \mathscr{L}^{\mathbf{\Delta}}(R)$ for every $x \in M$.

Proof. If $N \subset^{\prime} M$ and $x \in M$, and if $A \cap x^{-1} N=0$ for some $A \in \mathfrak{L}(R)$, then $x A \cap N=0, x A=0$, and $A \subset x^{-1} N$. Thus $A=0$, and we conclude that $x^{-1} N$ $\in \mathcal{L}^{\mathbf{\Lambda}}(R)$.

Conversely, if $K \in \mathfrak{M}(M)$ and $K \cap N=0$, and if $x^{-1} N \in \mathscr{L}^{\mathbf{\Lambda}}(R)$ for every $x \in K$, then $x\left(x^{-1} N\right)=0$ and $x \in M^{\mathbf{\Delta}}$. Hence $K=0$ and $N C^{\prime} M$.

6.2. Theorem. If $\left\{N_{i}\right\} \subset \mathfrak{T C}(M)$ and, for each $i, K_{i}$ is selected so that $N_{i} \subset^{\prime} K_{i}$, then $\cup_{i} N_{i} \subset^{\prime} \bigcup_{i} K_{i}$.

Proof. If $P \in \mathscr{T T}(M)$ such that $P \subset \bigcup_{i} K_{i}$ and $P \cap\left(\bigcup_{i} N_{i}\right)=0$, then each $k \in P$ has the form $k=\sum_{i} k_{i}, k_{i} \in K_{i}$. If $A=\bigcap_{i} k_{i}^{-1} N_{i}$ (a finite intersection), then $A \in \mathscr{L}^{\wedge}(R)$ by 6.1 and $k A=0$. Hence $k=0$, and $P=0$. This proves 6.2.

6.3. Theorem. If $N \in M(M)$ and $x \in M$ such that $x^{-1} N \in \mathscr{L}^{\wedge}(R)$, then $N \subset^{\prime} N+(x)$.

Proof. Since $(y+x r)^{-1} N=(x r)^{-1} N=r^{-1}\left(x^{-1} N\right) \in \mathcal{L}^{\wedge}(R)$ for every $y \in N$ and $r \in R$ (and similarly for $(y+n x)^{-1} N, n$ an integer), 6.3 follows from 6.1.

6.4. THEOREM. Each $N \in \mathbb{T}(M)$ has a unique maximal essential extension $N^{t}$ given by $N^{t}=\left\{x ; x \in M, x^{-1} N \in \mathscr{L}^{\mathbf{\Lambda}}(R)\right\}$.

Proof. The union of all essential extensions of $N$ is the unique maximal essential extension by 6.2. The rest of the theorem follows from 6.3.

This theorem applies equally well to $R$. Thus each $A \in \mathcal{L}(R)$ has a unique maximal essential extension $A^{s}=\left\{r ; r \in R, r^{-1} A \in \mathcal{L}^{\mathbf{\Lambda}}(R)\right\}$.

That 6.4 is not true in general may be seen from the example that follows.

6.5. Example. Let $N C^{\prime} M$ and $P=M \oplus(M-N)$. Define the submodule $K$ of $P$ as follows: $K=\{(x, x+N) ; x \in M\}$. Then both $M$ and $K$ are maximal essential extensions of $N$ in $P$. If $M^{\boldsymbol{\Lambda}}=0$, then $P^{\boldsymbol{\Lambda}}=M-N$.

6.6. TheOREM. $s \in C_{m}^{\prime 0}(\mathfrak{L}) / p$ and $t \in C_{m}^{0}(\mathfrak{T}) / p$.

Proof. If $A, B \in \mathscr{T}(M), A \subset B$, then $A+B C^{\prime} A^{t}+B^{t}$ by 6.2. Thus $B C^{\prime} A^{t}+B^{t}$ and $A^{t} \subset B^{t}$. Therefore it is clear that $t \in C^{0}(\mathfrak{T})$. Since $A \cap B$ $C^{\prime} A^{t} \cap B^{t} C^{\prime}(A \cap B)^{t}$ and $(A \cap B)^{t} C A^{t} \cap B^{t}$, we have $(A \cap B)^{t}=A^{t} \cap B^{t}$ and $t \in C_{m}^{0}(\mathfrak{N})$. Clearly each $A^{t}$ is prime, and hence $t \geqq p$.

We need only prove that $s \in C^{\prime}(\mathscr{L})$ to complete the proof of 6.6. Let us actually prove the stronger result that $s x^{-1}=x^{-1} t$ for every $x \in M$. Let $A \in \mathscr{L}(R)$ with $A \neq 0, x \in M$ and $N \in \mathscr{M}(M)$ such that $A \subset x^{-1} N^{t}$. Then $x A \subset N^{t}$ and either $x A=0$ or $x A \cap N \neq 0$. In either case, we have $A \cap x^{-1} N \neq 0$. Thus $x^{-1} N \subset^{\prime} x^{-1} N^{t}$ and therefore $x^{-1} N^{t} C^{\prime}\left(x^{-1} N\right)^{8}$. On the other hand, if 
$r \in\left(x^{-1} N\right)^{s}$ and $A=r^{-1}\left(x^{-1} N\right)$, then $A \in \mathscr{L}^{\wedge}(R)$. Hence $x r A \subset N, x r \in N^{t}$ and $r \in x^{-1} N^{t}$. Thus $\left(x^{-1} N\right)^{s} \subset x^{-1} N^{t}$ and, in view of the earlier inclusion, $\left(x^{-1} N\right)^{s}$ $=x^{-1} N^{t}$. This completes the proof of 6.6 .

It is clear now that the assumptions $R^{\mathbf{\Delta}}=0$ and $M^{\mathbf{\Delta}}=0$ lead to realizations of the closure operations $s$ and $t$ discussed in $\$ 4$. Actually, the closure operations $s$ and $t$ are reducible in the sense of (FI, $\$ 6)$. Thus, for each $A \in \mathcal{L}^{\text {s }}(R)$, $A \neq R$, there exists some nonzero $B \in \mathcal{L}^{\prime}(R)$ such that $A \cap B=0$, and similarly for $t$. Hence $\mathscr{L}^{s}(R)$ and $\mathfrak{T}^{t}(M)$ are complemented modular lattices by (FI, 6.2).

Let us now show that the complete Boolean algebra $\mathfrak{M C}^{\circ}(M)(=\mathfrak{H C}(\mathfrak{T}))$ is a sublattice of $\mathfrak{T}^{t}(M)$. Analogous remarks will hold for $\mathfrak{L}^{f}(R)$ in $\mathfrak{L}^{s}(R)$.

If $\left\{A_{i}\right\} \subset \mathcal{L}^{f}(R)$ and $A=\bigcap_{i} A_{i}$, then by definition $\left(\cup_{i}{ }^{l} A_{i}\right)^{g}={ }^{l} A$. For every $x \in \in^{l} A, x^{-1}\left(\bigcup_{i}{ }^{l} A_{i}\right) \supset A \cup\left(\bigcup_{i} A_{i}^{l}\right) \in \mathscr{L}^{\Delta}(R)$. Hence $\bigcup_{i}{ }^{l} A_{i} C^{\prime l} A$ by 6.1 , and $\left(\mathrm{U}_{i}{ }^{l} A_{i}\right)^{t}={ }^{l} A$. Thus $\mathfrak{m}^{\circ}(M)$ is a sublattice of $\mathfrak{T}^{t}(M)$. We shall presently prove that $\mathfrak{T}^{o}(M)$ is the center of $\mathfrak{T}^{t}(M)$. First, let us prove the corresponding result for $R$.

6.7. Theorem. The Boolean algebra $\mathfrak{L}^{f}(R)$ is the center of $\mathscr{L}^{s}(R)$.

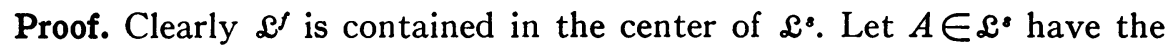
unique complement $A^{\prime}$, so that $A$ is also the unique complement of $A^{\prime}$. We shall first prove that $A, A^{\prime} \in \mathcal{L}^{\iota}$. This will be done by showing that $r A \cap A^{\prime}=0$ for every $r \in R$, which will imply that $r A \subset A$ for every $r \in R$. If $r a \in A^{\prime}$ for some $r \in R$ and $a \in A$, then $(r a+a) R \cap A^{\prime}=0$ since $r a x+a x \in A^{\prime}$ implies $a x \in A^{\prime}$ and $a x=r a x=0$. Hence $(r a+a) R \subset A, r a R \subset A$, and $r a \in A$. Since $r a \in A^{\prime}$ by assumption, $r a=0$. This proves that $A$ is an ideal of $R$. Similarly, $A^{\prime} \in \mathcal{L}^{\prime}$.

If $r A=0$ for some $r \in A$, then $r\left(A+A^{\prime}\right)=0$ and $r=0$; hence $A \in \mathscr{F}(R)$. Since $A^{f}=A^{s}$ for $A \in \mathcal{F}(R), A \in \mathcal{L}^{f}(R)$ and 6.7 follows.

6.8. TheOREM. Let $N \subset^{\prime} M$ and, for every $K \in \Re(N), K^{u}$ be the maximal essential extension of $K$ in $N$. Then $(K \cap N)^{u}=K^{t} \cap N, K \in \mathfrak{M}(M)$, and $\mathfrak{N}^{t}(M) \cong \mathfrak{N}^{u}(N)$ under the correspondence $K \rightarrow K \cap N, K \in \mathfrak{T}^{t}(M)$.

Proof. If $x \in(K \cap N)^{u}$, then $x \in N$ and $x^{-1}(K \cap N) \in \mathscr{L}^{\Delta}(R)$. Hence $x^{-1} K$ $\in \mathscr{L}^{\wedge}(R)$ and $x \in K^{t}$. On the other hand, if $x \in K^{t} \cap N$, then $x \in N$ and $x^{-1} K \in \mathscr{L}^{\wedge}(R)$. Hence $x^{-1}(K \cap N) \in \mathscr{L}^{\wedge}(R)$ and $x \in(K \cap N)^{u}$. This proves that $(K \cap N)^{u}=K^{t} \cap N$ for every $K \in \mathfrak{M}(M)$.

For $K_{i} \in \mathfrak{T}(M),\left(K_{1} \cap N\right)^{u}=\left(K_{2} \cap N\right)^{u}$ implies $\left(K_{1}^{t} \cap N\right)^{t}=\left(K_{2}^{t} \cap N\right)^{t}$ and $K_{1}^{t}=K_{2}^{t}$. Clearly $\left(K_{1} \cap N\right)^{u} \subset\left(K_{2} \cap N\right)^{u}$ if and only if $K_{1}^{t} \subset K_{2}^{t}$. This proves 6.8.

If $N \in \Re(M)$ and $N^{\prime}$ is a complement of $N$, then for every $K \supset N$ such that $K \cap N^{\prime}=0$, necessarily $N C^{\prime} K(\mathrm{FI}, \S 6)$.

If $K, N \in \mathscr{T}^{t}(M)$ and $K \nsubseteq N$, then $K$ is not an essential extension of $K \cap N$. Thus there exists a nonzero $A \in \mathfrak{M}(M)$ such that $A \subset K, A \cap N=0$.

We shall further restrict ourselves in the remainder of this section by as- 
suming that $s$ is homogeneous. By 4.6, it is clear that $t$ also is homogeneous. In view of our remarks of the previous paragraph, if $K$ and $N$ are distinct elements of $\mathfrak{T}^{t}(M)$, there exists an atom of $\mathfrak{T}^{t}(M)$ contained in one and only one of $K$ and $N$.

The following theorem was proved for prime rings in $[7,4.11]$.

6.9. THEOREM. If $x \in M, x \neq 0$, then $(x R)^{t}$ is an atom of $\mathfrak{M}^{t}(M)$ if and only if $x^{-1} 0$ is a maximal element of $\mathfrak{L}^{8}(R)$.

Proof. If $(x R)^{t}$ is an atom of $\Re^{t}(M)$ and $x^{-1} 0$ is not maximal in $\mathfrak{L}^{s}(R)$, then $x^{-1} 0 \subset C \subset R$ for some $C \in \mathcal{L}^{s}, C \neq x^{-1} 0, C \neq R$. Hence there exist atoms $A, B \in \mathcal{L}^{s}(R)$ for which $x^{-1} 0 \cap A=0, A \subset C$, and $B \cap C=0$. Evidently $x A \neq 0$ and $x B \neq 0$, and therefore $x A \cap x B \neq 0$ since $(x R)^{t}$ is an atom. Hence $x^{-1} 0$ $\cap(A+B) \neq 0$, contrary to the choice of $A$ and $B$. Thus $x^{-1} 0$ must be maximal in $\mathscr{L}^{8}$.

Conversely, if $x^{-1} 0$ is maximal in $\mathscr{L}^{s}$ and $N$ is an atom of $\mathbb{T}^{t}$ such that $N \subset(x R)^{t}$, then $N \cap x R \neq 0, x^{-1} N \supset x^{-1} 0$, and therefore $x^{-1} N=R$. Hence $x \in N$ and $(x R)^{t}$ is an atom of $\pi^{t}$.

From this theorem, $R$ cannot be an integral domain unless $\mathscr{L}^{s}(R)=\{0, R\}$. And if $R$ is an integral domain, our assumptions are such as to insure that $R$ necessarily has a right quotient division ring.

The atoms of $\mathfrak{T C}^{g}(M)$ may be characterized by use of the concept of perspectivity. Two elements $A$ and $B$ of $\mathfrak{T}^{t}(M)$ are called perspective [2, p. 118], and we write $A \sim B$, if and only if $A$ and $B$ have a common complement. It is easily shown that perspectivity is an equivalence relation on $\mathfrak{B}_{t}$, the set of all atoms of $\Re^{t}(M)$. As in [2, p. 120, Lemma 3], we may show that two distinct elements of $\mathfrak{B}_{t}$ are perspective if and only if their union contains a third atom. A ring-theoretic version of this result is as follows.

6.10. Theorem. For $A, B \in \mathfrak{B}_{t}, A \sim B$ if and only if there exist nonzero $a \in A$ and $b \in B$ such that $a^{-1} 0=b^{-1} 0$.

Proof. The theorem is obvious if $A=B$, so let us assume that $A \neq B$. If $a^{-1} 0=b^{-1} 0$ for some nonzero $a \in A$ and $b \in B$, let $C=[(a+b) R] t$. Then $C$ is an atom by 6.9, $C \subset A \vee B$, and $A \sim B$.

Conversely, if $A \sim B$, select $C \subset A \vee B, C \neq A$ and $C \neq B$. Each nonzero $c \in C$ has the form $c=a+b$ for some nonzero $a \in A$ and $b \in B$. Since $c^{-1} 0$ is a maximal element of $\mathscr{L}^{s}$ and $c^{-1} 0=a^{-1} 0 \cap b^{-1} 0$, we have $a^{-1} 0=b^{-1} 0$. This proves 6.10 .

Our remarks on perspective elements apply equally well to $\mathfrak{L}^{8}(R)$. The relations of perspectivity on $\mathfrak{B}_{s}$ and $\mathfrak{B}_{t}$ are connected by the following result.

6.11. Theorem. If $A, B \in B_{\text {s }}$ and $x, y \in M$ with $x A \neq 0$ and $y B \neq 0$, then $A \sim B$ if and only if $(x A)^{t} \sim(y B)^{t}$.

Proof. If $A \sim B$, so that $a^{-1} 0=b^{-1} 0$ for some nonzero $a \in A$ and $b \in R$ then 
$(x a)^{-1} 0=(y b)^{-1} 0$ and $(x A)^{t} \sim(y B)^{t}$. Conversely, if $(x A)^{t} \sim(y B)^{t}$, there exist nonzero $u \in(x A)^{t}$ and $v \in(y B)^{t}$ such that $u^{-1} 0=v^{-1} 0$. Since $u R \cap x A \neq 0$, $u r=x a \neq 0$ for some $r \in R$ and $a \in A$, and $(x a)^{-1} 0=(v r)^{-1} 0$. Similarly, $v r r^{\prime}$ $=y b \neq 0$ for some $r^{\prime} \in R$ and $b \in B$, and $\left(x a r^{\prime}\right)^{-1} 0=(y b)^{-1} 0$. Since $A \cap x^{-1} 0$ $=B \cap y^{-1} 0=0$, evidently $\left(a r^{\prime}\right)^{-1} 0=b^{-1} 0$ and $A \sim B$.

For each $A \in \mathfrak{B}_{t}$, let $A^{*}$ designate the union in $\mathfrak{T T}^{t}(M)$ of all $B \sim A$. It is easily demonstrated that an atom $B \subset A^{*}$ if and only if $B \sim A$.

\subsection{Theorem. If $A \in \mathfrak{B}_{t}$, then $A^{*}$ is an atom of the center of $\mathfrak{T H}^{t}(M)$.}

Proof. If $B$ is a complement of $A^{*}$ and $B$ is not unique, then there exists some atom $C$ such that $C \cap A^{*}=C \cap B=0$. Since $C \cap\left(A^{*}+B\right) \neq 0, c=a+b$ for some nonzero $a \in A, b \in B, c \in C$. Clearly $a^{-1} 0=b^{-1} 0=c^{-1} 0, C \sim(a R)^{t}$, and $C \sim A$ contrary to assumption. Hence $A^{*}$ has a unique complement and is in the center of $\boldsymbol{M}^{t}$.

If $N$ is an atom of the center of $\mathfrak{T}^{t}$ and $A \subset N, A \in \mathfrak{B}_{t}$, then $N \subset A^{*}$. If $N \neq A^{*}$, there exists $B \in \mathfrak{B}_{\ell}$ such that $B \subset A^{*}$ and $B \cap N=0$. Since $A \sim B$, $C \subset A \vee B$ for some atom $C$ distinct from $A$ and $B$. If $C \subset N$, then $C \vee A \subset N$ and $B C N$ contrary to our choice of $B$. If $C \subset N^{\prime}$, the complement of $N$, then $C \vee B \subset N^{\prime}$ and $A \subset N^{\prime}$ contrary to our choice of $A$. Hence $N=A^{*}$, and 6.12 is proved.

Letting $M=R^{+}$in the theorem above, we have that the atoms of $\mathscr{L}^{f}(R)$, the center of $\mathscr{L}^{s}(R)$, are of the form $A^{*}$ for $A \in \mathfrak{B}_{s}$. This is in keeping with the classical result for a ring $R$ with minimal right ideals, in which case each foot of the socle is a union of isomorphic (as $R$-modules) minimal right ideals.

If $A^{*}$ is an atom of $\mathscr{L}^{f}(R), A \in \mathfrak{B}_{s}$, then $\left(M A^{*}\right)^{t}$ is in $\mathfrak{T}^{g}(M)$ and hence in the center of $\mathfrak{N}^{t}(M)$. Let $N$ be an atom of the center of $\mathfrak{T}^{t}, N \subset\left(M A^{*}\right)^{t}$. If $x \in M A^{*}, x \neq 0$, and $B \in \mathfrak{B}_{s}$ with $x B \neq 0$, then necessarily $B \subset A^{*}$. Since $N A^{*} \neq 0, y C \neq 0$ for some $y \in N$ and $C \sim B$. Thus $(x B)^{t} \sim(y C)^{t}$ by 6.11 , and $x B \subset N$ by 6.12 . Hence $x B \subset N$ for every atom $B$ of $\mathscr{L}^{s}$ and $x^{-1} N \in \mathscr{L}^{\wedge}$. Therefore $x \in N^{t}=N$, and $\left(M A^{*}\right)=N$. We have proved that the atoms of the center of $\mathfrak{T}^{t}$ are just the atoms of $\mathfrak{N}^{0}$. Each element of the center of $\mathfrak{N}^{t}$ is a union of atoms of the center, and the following theorem is established.

\subsection{Theorem. The Boolean algebra $\mathfrak{T}^{\circ}(M)$ is the center of $\mathfrak{T C}^{t}(M)$.}

The well-known result that every complete atomic modular lattice is a discrete direct sum of atomic projective geometries $[2 ;$ p. 131] applies to the lattice $\Re^{t}(M)$. In our case, the projective geometries are the submodules $N_{i}^{*}$ where $N_{i} \in \mathscr{B}_{t}$, and $M=\mathrm{V}_{i} N_{i}^{*}$.

7. Injective modules. The $R$-module $M$ is called injective [4] if and only if for every pair $A, B$ of $R$-modules with $A \subset B$, each homomorphism of $A$ into $M$ can be extended to one of $B$ into $M$. If $R$ is a ring with unity 1 and if 1 is the identity operator of an $R$-module $M$, then it has been shown by Baer [1] and more recently by Eckmann and Schopf [4] that $M$ has a mininal injective 
extension $\hat{M}$, unique up to an isomorphism. At the same time, $\hat{M}$ is a maximal essential extension of $M$. These results are true for any ring $R$ and any $R$ module, as we shall now prove.

7.1. THEOREM. If $R$ is a ring and $M$ is an $R$-module, there exists a minimal injective extension $\hat{M}$ of $M$ that is unique up to an isomorphism.

Proof. We may imbed $R$ in a ring $S$ with unity in a standard way. Thus let $S=\{(a, n) ; a \in R, n \in I\}$, where $I$ is the ring of integers, with the operations defined as if $(a, n)=a+n[12$, p. 87]. If we identify $R$ with the subring $\{(a, 0) ; a \in R\}$ of $S$, then $R$ is an ideal of $S$.

Now $M$ becomes an $S$-module if we define $x(a, n)=x a+n x$ for each $x \in M$ and $(a, n) \in S$. The identity element $(0,1)$ of $S$ acts as the identity operator on $M$. Therefore, as an $S$-module, $M$ has a minimal injective extension $\hat{M}$.

In order to prove that $\hat{M}$ is an injective extension of $M$ as an $R$-module, let $A$ and $B$ be $R$-modules with $A \subset B$ and let $\phi$ be a $R$-homomorphism of $A$ into $\hat{M}$. Then $\phi$ also is an $S$-homomorphism of $A$ into $\hat{M}$ if we define $\phi[x(a, n)]$ $=\phi(x a+n x), x \in A,(a, n) \in S$. Thus $\phi$ can be extended to an $S$-homomorphism $\phi^{\prime}$ of $B$ into $\hat{M}$. Clearly $\phi^{\prime}$ is an $R$-homomorphism of $B$ into $\hat{M}$, and we conclude that $M$ is an injective extension of the $R$-module $M$.

Each maximal essential extension of $M$ in $\hat{M}$ will be a minimal injective extension of $M$. The uniqueness of this minimal injective extension may be shown as in [4].

Let us assume henceforth in this section that $R$ is a ring and $M$ is an $R$ module such that

$$
R^{\mathbf{\Lambda}}=0 \text { and } M^{\boldsymbol{\Lambda}}=0 .
$$

Again, $\hat{M}$ designates the minimal injective extension of $M$. We know that $\hat{M}^{\mathbf{\Lambda}}=0$. For $N$ in $\mathfrak{M}(\hat{M})(\mathfrak{T}(M))$, let $N^{v}\left(N^{t}\right)$ be the maximal essential extension of $N$ in $\hat{M}(M)$. According to $6.8, \mathfrak{N}^{v}(\hat{M}) \cong \mathfrak{M}^{t}(M)$ under the correspondence $N \rightarrow N \cap M, N \in \mathscr{M}^{v}(\hat{M})$. For each $N \in \mathscr{M}(\hat{M}), N^{v}$ is also the minimal injective extension of $N$.

7.2. Lemma. If $N_{i} \in \mathscr{T r}^{\mathfrak{v}}(\hat{M})$, then $N_{1} \bigvee N_{2}=N_{1}+N_{2}$.

Proof. The lemma is obvious if $N_{1} \subset N_{2}$, so let us assume $N_{1} \llbracket N_{2}$. Select $K \in \mathfrak{M}(\hat{M})$ maximal so that $K \subset N_{1}, K \cap N_{2}=0$. Clearly $K \in \mathscr{N}^{v}(\hat{M})$ and $N_{1}=\left(N_{1} \cap N_{2}\right) \oplus K$; for $N_{1}-K$ is an essential extension of $N_{1} \cap N_{2}$, and therefore $N_{1}-K \cong N_{1} \cap N_{2}$. Since $N_{2}$ and $K$ are injective, so is $N_{2} \oplus K$. Thus $N_{1}+N_{2}=N_{2} \oplus K$, and evidently $N_{1} \bigvee N_{2}=N_{1}+N_{2}$.

We shall designate by $\mathcal{C}(\hat{M}, R)$ the centralizer of $R$ over $\hat{M}$, and we shall consider $\hat{M}$ as a $(\mathcal{C}, R)$-module.

7.3. Theorem. For each $\alpha \in \mathfrak{C}(\hat{M}, R), \alpha^{-1} v=v \alpha^{-1}$.

Proof. If $N \in \mathscr{M}(\hat{M})$ and $x \in\left(\alpha^{-1} N\right)^{v}$, then $x^{-1}\left(\alpha^{-1} N\right)=(\alpha x)^{-1} N \in \mathcal{L}^{\Lambda}(R)$, 
$\alpha x \in N^{v}$, and finally $x \in \alpha^{-1} N^{v}$. Thus $\left(\alpha^{-1} N\right)^{v} \subset \alpha^{-1} N^{v}$. Similarly, one proves that $\alpha^{-1} N^{v} C\left(\alpha^{-1} N\right)^{v}$, and 7.3 follows.

If $N \in M^{\wedge}(M)$ and $\alpha$ is a homomorphism of the $R$-module $N$ into $M$, then there exists a unique element $\beta \in \mathrm{C}(\hat{M}, R)$ such that $\beta x=\alpha x$ for every $x \in N$. Thus, if there were two elements of $\mathfrak{e}$ agreeing with $\alpha$ on $N$, their difference $\gamma$ would annihilate $N, \gamma^{-1} 0 \supset N$. Since $\gamma^{-1} 0 \in \mathscr{N}^{v}(\hat{M})$ by $7.3, \gamma^{-1} 0=\hat{M}$ and $\gamma=0$. From these remarks, it is evident that $\mathfrak{e}(\hat{M}, R)$ is isomorphic to the extended centralizer of $R$ over $M$ as defined in [6]. Thus the following results on $\mathfrak{C}(\hat{M}, R)$ are generalizations of some of our previous results on the extended centralizer $[6 ; 7]$.

For each $N \in \mathfrak{M}(\hat{M})$, let

$$
\mathfrak{e}(N)=\{\alpha ; \alpha \in \mathfrak{C}(\hat{M}, R), \alpha \hat{M} \subset N\} .
$$

As in $[7,5.5]$, the principal right ideals of the regular ring $\mathfrak{e}(\hat{M}, R)$ may be identified as follows.

7.4. Lemma. If $A \in \mathfrak{L}(\mathfrak{C}), A$ is principal if and only if $A=\mathfrak{C}(N)$ for some $N \in \mathbb{N}^{v}(\hat{M})$.

Proof. If $N \in \mathscr{T}^{v}(\hat{M})$ and $N^{\prime}$ is a complement of $N$, then $N+N^{\prime}=\hat{M}$. Let $\epsilon \in \mathcal{C}$ be defined as follows: $\epsilon x=x, x \in N ; \epsilon N^{\prime}=0$. Then $(\epsilon \mathcal{C}) \hat{M}=N$. If $\alpha \hat{M} \subset N$ for $\alpha \in \mathfrak{C}$, then $\epsilon \alpha=\alpha$ and $\alpha \in \epsilon \mathfrak{C}$. Therefore $\mathfrak{C}(N)=\epsilon \mathfrak{C}$.

Conversely, if $A=\alpha \mathfrak{C}$ for some $\alpha \in \mathfrak{C}$ and $N=\alpha \hat{M}$, then $N \in \mathscr{M}^{v}(\hat{M})$ by 7.3. Clearly $A=\mathfrak{e}(N)$, and the lemma is proved.

7.5. TheOREM. The lattice $\mathfrak{T}^{t}(M)$ is isomorphic to the lattice $P(\mathfrak{C})$ of principal right ideals of $\mathfrak{e}(\hat{M}, R)$ under the correspondence $N \rightarrow \mathfrak{C}\left(N^{v}\right), N \in \mathfrak{M}^{t}(M)$.

Proof. We shall prove that $\mathfrak{T}^{v}(\hat{M}) \cong \mathcal{P}(\mathcal{C})$, which will prove our theorem in view of 6.8. Evidently if $K, N \in \mathscr{T}^{v}$ with $K \subset N$, then $\mathfrak{e}(K) \subset \mathfrak{e}(N)$. If $K \neq N$ and $\mathfrak{e}(N)=\alpha \mathfrak{e}$, then $\alpha \hat{M}=N$ and $\alpha \notin \mathfrak{e}(K)$; thus $\mathfrak{e}(K) \neq \mathfrak{e}(N)$. On the other hand, if $\alpha, \beta \in \mathfrak{C}$ and $\alpha \mathfrak{e} \subset \beta \mathfrak{e}$, then $\alpha=\beta \gamma$ for some $\gamma \in \mathfrak{C}$ and $\alpha \hat{M} \subset \beta \hat{M}$; also, $\alpha \hat{M} \neq \beta \hat{M}$ if $\alpha \mathfrak{C} \neq \beta \mathbb{C}$. This proves 7.5.

The center of $\mathfrak{T}^{v}(\hat{M})$ may be identified as follows:

7.6. ThEOREM. If $N \in \mathfrak{M}^{v}(\hat{M}), N$ is in the center of $\mathfrak{M}^{\circ}$ if and only if $\mathfrak{e} N \subset N$.

Proof. Assume that $\alpha \in \mathcal{C}$ and $x \in N$ such that $\alpha x \notin N$. Since $(\alpha x)^{-1} N$ $\bigoplus_{\mathscr{L}^{\wedge}}(R)$, there exists $A \in \mathfrak{L}(R)$ such that $(\alpha x) A \neq 0,(\alpha x) A \cap N=0$. Let $N^{\prime}$ be a complement of $N$ containing $(\alpha x) A$. Clearly $(x-\alpha x) A \cap N=0$ and $(x-\alpha x) A \cap N^{\prime}=0$. Thus, since $(x-\alpha x) A \neq 0, N$ has another complement $N^{\prime \prime}$ containing $(x-\alpha x) A$. Consequently $N$ is not in the center of $\mathfrak{T}^{v}(\hat{M})$.

Conversely, if $N$ is not in the center of $\mathfrak{T}^{v}(\hat{M})$ and $N^{\prime}$ is some complement of $N$, then $N^{\prime}$ will in turn have a complement $N^{\prime \prime} \neq N$. Let $\epsilon \in \mathcal{C}$ be defined by: $\epsilon x=x, x \in N^{\prime \prime} ; \epsilon x=0, x \in N^{\prime}$. Now each $x \in N$ has the form $x=x^{\prime}+x^{\prime \prime}, x^{\prime} \in N^{\prime}$, 
$x^{\prime \prime} \in N^{\prime \prime}$, and for some $x \in N, x^{\prime \prime} \notin N$. Hence $\epsilon N \nsubseteq N$. This proves 7.6.

7.7. THEOREM. If the closure operation $s$ is homogeneous and if $\left\{N_{i}\right\}$ is the set of atoms in the center of $\mathfrak{T}^{v}(\hat{M})$, then

$$
\mathfrak{C}(\hat{M}, R) \cong \sum_{i}^{*} \mathfrak{C}\left(N_{i}, R\right)
$$

Proof. If $N=\cup_{i} N_{i}$, then $N C^{\prime} \hat{M}$ so that each $R$-homomorphism of $N$ into $\hat{M}$ has a unique extension in $\mathfrak{e}(\hat{M}, R)$. Since $\mathfrak{e} N_{i} \subset N_{i}$ for each $i, \mathfrak{e} N \subset N$ and $\mathfrak{C}(\hat{M}, R) \cong \mathfrak{C}(N, R)$. Each $\alpha \in \mathfrak{C}(N, R)$ has the form $\alpha=\sum_{i}^{*} \alpha_{i}$ where $\alpha_{i}$ $\in \mathfrak{C}\left(N_{i}, R\right)$. Thus $\mathfrak{e}(N, R) \cong \sum_{i}^{*} \mathfrak{e}\left(N_{i}, R\right)$ and the theorem is proved.

It is easily shown that each $\mathfrak{C}\left(N_{i}, R\right)$ of the theorem above is a primitive ring with minimal right ideals. In case $M=R^{+}, \mathfrak{e}(\hat{M}, R)$ is known to be the maximal right quotient ring of $R[6$, p. 895]. Thus, if $s$ is homogeneous, $R$ has as maximal right quotient ring a full direct sum of primitive rings with minimal right ideals.

Smith College, Northampton, Mass. 